\title{
INTELLECTUAL PROPERTY RIGHTS PROTECTION AND ENFORCEMENT IN A SOFTWARE DUOPOLY
}

\author{
Jiří Střelický \\ Krešimir Žigić
}
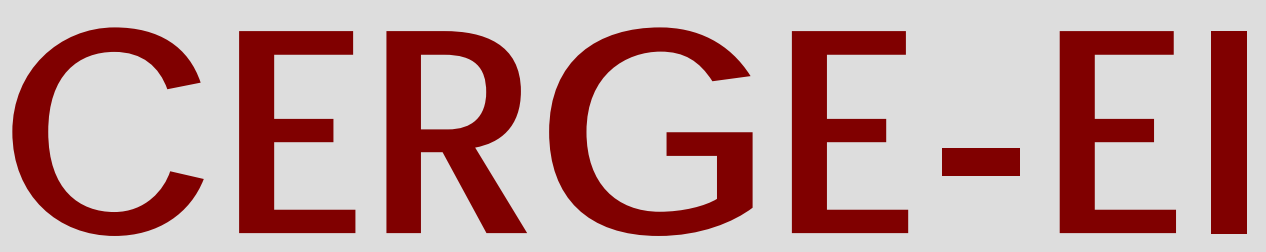

Charles University Centerfor Economic Research and Graduate Education Academy of Sciences of the Czech Republic Ec onomic s Institute 


\title{
Working Paper Series 435 (ISSN 1211-3298)
}

\section{Intellectual Property Rights Protection and Enforcement in a Software Duopoly}

\author{
Jiří Střelický \\ Krešimir Žigić
}

CERGE-EI

Prague, March 2011 
ISBN 978-80-7343-236-2 (Univerzita Karlova. Centrum pro ekonomický výzkum a doktorské studium)

ISBN 978-80-7344-226-2 (Národohospodářský ústav AV ČR, v.v.i.) 


\title{
Intellectual Property Rights Protection and Enforcement in a Software Duopoly ${ }^{1}$
}

\author{
Jiří Střelický ${ }^{2}$ and Krešimir Žigić ${ }^{3}$
}

\begin{abstract}
We study the economic impacts of the interaction between a regulator's Intellectual Property Rights (IPR) protection policy against software piracy on the one side and the forms of IPR protection that software producers may themselves undertake to protect their intellectual property on the other side. Two developers, each offering a variety of different quality, compete for heterogeneous users who choose among purchasing a legal version, using an illegal copy, and not using a product at all. Using an illegal version violates IPR and is thus punishable when disclosed. If a developer considers the level of piracy as high, he can either introduce a form of physical protection for his product or introduce a protection in the form of restricting support and other services to illegal users. The quality of each developer's product is exogenously given, and the developers compete in prices. We examine the above issues within the framework of Bertrand and Stackelberg competition while the monopoly set-up serves as a point of reference.
\end{abstract}

\begin{abstract}
Abstrakt
V našem článku se zabýváme interakcí mezi ochranou duševních práv (IPR) prováděnou regulátorem a mezi ochranou díla proti kopírování, která je implementována softwarovou firmou, a to vše analyzujeme v závislosti na zvoleném zpo̊sobu ochrany. Na trhu jsou dvě softwarové firmy, které nabízejí software rozdílné kvality, a soutěží o heterogenní uživatele, kteří si vybírají mezi legálním používáním softwaru, nelegálním užitím a celkovým nepoužíváním. Používání nelegální verze porušuje ochranu duševních práv a v případě odhalení následuje pokuta. Pokud softwarová firma vnímá úroveň softwarového pirátství na trhu jako př́liš velkou, může zavést vlastní formu ochrany svých produktů nebo může zavést omezení nelegálním uživatelům na své podpưrné služby. Kvalita produktů od každé softwarové firmy je exogenně dána a firmy proti sobě soutěží pouze cenami. V článku rozebíráme výše zmiňovaný koncept v Bertrand a Stackelberg soutěži, zatímco koncept monopolu slouží jen k porovnání.
\end{abstract}

Keywords: Vertically differentiated duopoly, Software piracy, Bertrand competition, Private and public intellectual property rights protection.

JEL Classification: D43, L11, L21, O25, O34.

\footnotetext{
${ }^{*}$ CERGE-EI is a joint workplace of the Center for Economic Research and Graduate Education, Charles University, and the Economics Institute of Academy of Sciences of the Czech Republic.

Address: CERGE-EI, P.O. Box 882, Politických vězňu 7, Prague 1, 111 21, Czech Republic.

${ }^{1}$ Authors would like to thank to Martin Peitz, Paul Belleflamme, Milan Horniaček, Levent Celik and Avner Shaked for their valuable comments.

2 jiri.strelicky@seznam.cz

${ }^{3}$ kresimir.zigic@cerge-ei.cz
} 


\section{Introduction}

During the last two decades violating Intellectual Property Rights (IPR) emerged as an important and hot economic and political issue since most of the world brands face a problem of illegal imitation of their products. The violation of IPR poses a threat to a wide range of products - from fashion such as Etro or Vuitton to Intel chipsets or Yamaha motorbikes. According to the WCO (World Customs Organization), 7\% (\$512 billion) of world trade takes place with fake merchandise ${ }^{4}$ and most customers buy fake products unknowingly.

Barely anybody in Moscow believes that a $\$ 20$ Rolex watch from a stallholder is an authentic one, but, on the other hand, hardly anyone assumes that a drug at a pharmacy, or car spare parts in dealer service centers are fake. In some cases, even professionals have difficulties to identify a particular product as a fake.

The key factor contributing to the creation of illegal imitations are low costs and low technical requirements. Based on such a view, the natural leaders among illegal imitations are "information" products or what are known as digital content products - software, movies, music, or e-books ${ }^{5}$. These products have two idiosyncratic attributes: imitations are $100 \%$ identical to the original and costs of copying are negligible. According to the report of the Business Software Alliance, the share of software that is pirated climbed to $41 \%$ of total units installed in 2008 and the global loss exceeded $\$ 50$ billion $^{6}$. Even in the US, where the rate of illegal usage is the lowest, it amounts to 20\%, while in Western Europe about one-third of installed software is used illegally. The top of the list with $80 \%$ and more of illegal software installed is occupied by Georgia, Pakistan, Indonesia, and China ${ }^{7}$.

The expansion of DVD burners accompanied with the penetration of broadband internet does not only increase the opportunity for illegal copying ${ }^{8}$, but also eliminates mass illegal producers from market. Illegal copies are, nowadays typically made (installed) by the end users themselves who do it wittingly and only for themselves ${ }^{9}$. This attribute changes the

\footnotetext{
${ }^{4}$ Two thirds of illegal imitations come from China, the rest mainly from Ukraine, Russia, Vietnam, the Philippines, according to the WCO.

${ }^{5}$ For information about mp3, movie, or e-book protection and their illegal copying see www.ifpi.org, www.riaa.com, www.pro-music.org.

${ }^{6}$ According to the IDC Global Software Piracy Study.

${ }^{7}$ see also The Economist, May 16th, 2009.

${ }^{8}$ Most of the illegal copies of digital content are easily accessible using P2P networks (direct connect, torrent trackers) or data sharing (Rapidshare). Note that easy downloading could be accompanied by relatively complicated installation/usage of illegal versions.

${ }^{9}$ We omit in the essay the problem of the black market with DVDs/CDs or software in the suburbs. These
} 
essentials of the fight against IPR violation. While, say, in pharmaceuticals, luxury goods, or electronics markets, end users might be often perceived as victims, in "information" markets, end-users of illegal copies are predominantly the ones that actually carry out IPR violation ${ }^{10}$. Thus, the fight in digital content markets is now aimed mainly against end users (meaning both retail and corporate users ${ }^{11}$.

In this essay, we focus on such digital content markets (like the software market) where only the end users violate property rights. More specifically, we study the strategic interactions among software developers that may undertake various forms of product protections (developer IPR protection) and also analyze the impact of regulator (or government) IPR protection on such developer IPR protection. In particular, we put forward a dynamic twostage duopoly model, where the last stage competition is in prices, and where each developer competes for users with different price sensitivity on the same market. That is, we rely on a quality competition model (see, for instance, Shaked and Sutton, 1984, and Tirole, $1988^{12}$ ). In the first stage of the game, each developer has an option to choose a particular form of IPR protection. The government ${ }^{13}$ only sanctions illegal use of the product by means of imposing a penalty so those end users who illegally appropriate the software will be punished, if discovered.

We consider the two most common forms that the developers use to protect their products: a) decreasing product value to illegal users by, say, eliminating updates in antivirus or tune-up utilities ${ }^{14}$ and b) physical product protection by means of special CDs (or encryption kinds of piracy experienced a boom one decade ago and are now strongly declining especially in developed countries.

${ }^{10}$ However, companies try to distinguish between intentional piracy and the unconscious usage of an illegal version, e.g., Microsoft replaces fake versions with legal ones to users who bought a fake version of its software in good faith.

${ }^{11} \mathrm{~A}$ well-known examples aimed at end-users is suing students at US/EU universities for sharing software on university servers. Note that these actions are often accompanied with legal actions against the means of sharing e.g. closing Napster as the first famous case or the current hot suit against torrent tracker The Pirate Bay with the intention to close it.

${ }^{12}$ Shy (1999) addresses the same problem using a Hotelling-type spatial competition model.

${ }^{13}$ In the essay, we do not distinguish the role (objective) of government from the regulator's role or from the role of private authorities executing any monitoring as e.g. the RIAA (Recording Industry Association of America), the MPA (Motion Picture Association), the BSA (Business Software Alliance).

${ }^{14}$ Illegal versions of some antivirus software, e.g., Symantec Antivirus, do not update their installed databases of viruses and thus the PC is more vulnerable in the case of the latest virus attack, or tuneup utilities do not update their internal list of supported problems, so some new errors cannot be corrected. 
against cracking) like in games, where copies created on a standard DVD burner do not work anymore. These measures are known in the literature as "technical protection measures" which enhance copyright enforcement (see Scotchmer, 2004, for an excellent survey on this topic) and one of the most known ways of protecting the digital content is DRM (Digital Rights Management) system ${ }^{15}$.

To capture the regulator's role in a simple manner, we assume that imposing a penalty is the only instrument for reducing or eliminating the illegal usage of the product that is under copyright protection. The government's reliance on taxes and subsidies as an instrument of IPR protection are not considered very realistic in the given context and is thus assumed away in the further analysis. Moreover, in order to focus on the impact of the penalty in different market configurations and its interaction with the private developers' enforcement, we do not assign the regulator a particular objective function such as maximizing social welfare, but we rather look at the penalty as exogenous and discuss its impact on developer equilibrium values and on the developers' choice of the form of IPR protection.

In most countries, governments are responsible for the creation of a legal environment for IPR enforcement and prevention from piracy. Nevertheless, a government's objective does not in general coincide with the developers'. First, if the costs of copying are negligible, the more users use the product, the higher social welfare is. Moreover, the original product can be an inspiration for other developers and its wide spreading may raise further product development and consequently, social welfare. On the other hand, in an environment where a product could be freely copied, the producers' incentives to develop new products are suppressed $^{16}$. Thus, the regulator activity in setting IPR protection, exerting monitoring, and the scale of penalty for users convicted from illegal usage usually balances the trade-off between the dissemination of knowledge and products on the one side, and preserving the incentives to innovate on the other side. In setting the level of IPR protection, a government

\footnotetext{
${ }^{15} \mathrm{DRM}$ is an umbrella term for various technologies that limit the usage of digital content in an unintended way by the developer. DRM is used by a lot of major content providers such as Microsoft, Sony, Amazon, or Apple. DRM is sometimes considered a controversial approach to protecting the IPR since it often restricts the usage ways beyond the copyright laws (e.g., not only against illegal copying, but even the legal usage, such as using the legally bought e-book on only one device). DRM technologies, however, were effectively implemented in selected cases as, e.g., in the case of Apple (iTunes). Nowadays most content providers experiment with DRM-free alternatives mainly in music. In movies or e-books, DRM is still quite used. See Belleflamme and Peitz (2010).

${ }^{16}$ There are other effects, such as tax losses, raising unemployment etc., which could be studied separately.
} 
may favour one of the developers, e.g., in the case of a domestic dominant developer competing with a foreign developer, the government may, for instance, adapt IPR enforcement to favour the domestic developer or vice versa ${ }^{17}$. Thus, to introduce explicitly the regulator's objective function, we would have to put "more structure" into our model. As already noted, the choice of the optimal level of government IPR (or the optimal expected penalty) is out of the scope of our analysis. We, however, briefly discuss in the conclusion the possible extensions of our analysis to normative issues.

Since the legal environment as well as the regulator's activity are publicly observable, users can estimate the probability of being caught and then convicted for copyright violation and so correctly calculate the expected size of the penalty. Thus, if a user decides to use an illegal version, he can evaluate the expected penalty (EP), which can be considered as the cost of illegal usage.

The software market may distinguish itself from other digital content markets due to potentially high Network Effects (NEs) coming from software usage. NEs mean that increasing the base of users by, say, allowing the copying of a product to some other users, raises the utility of all users and thus adds extra value to the product. We, on the other hand, consider NE unimportant, but we nevertheless briefly discuss how NE can be easily incorporated in our set-up (see section 2.5 ).

It is important to stress at the outset that our approach is a bit different from the current literature on software piracy. To put our analysis into context, we follow the very recent comprehensive and influential survey of digital piracy by Belleflamme and Peitz, 2010 (see also Peitz and Waelbroeck, 2006). According to this, our approach belongs to the i) end-user piracy models that ii) includes the competitive effects meaning that there are two producers of substitutable and piratable digital products that directly compete with each other (see Belleflamme and Peitz, 2011, p. 20).

As clearly seen from the Belleflamme and Peitz (2011), there are indeed only a few articles that deal with the positive and normative issues of digital piracy while explicitly modeling direct firm competition. Moreover, all of these papers, in general, rely on the notion of horizontal product differentiation. The pioneering article seems to be the one of Shy and Thisse (1999), who analyze piracy in the Hotelling-type duopoly competition where users

\footnotetext{
${ }^{17}$ For illustration, we could use a comparison among countries that have strong developers (e.g., the US) and quite a severe protection of IPR with countries where no strong local developers exist, e.g., Finland, Sweden, or Norway, and their protection of IPR is moderate and more "open."
} 
have exogenous preferences for a particular developer. They show that a developer's decision to introduce protection against illegal copying depends mainly on the NEs, and that under strong NEs, each developer decides not to implement protection in order to make his software more attractive and to raise the users base. Jain (2008) builds upon the model of Shy and Thisse (1999) and assumes that firms can choose a level of IPR protection so that only a proportion of consumers with low product valuations (who are, by assumption, the only consumers interested in copying) can copy its product. In the absence of network effects, Jain shows that in such a set-up piracy can change the structure of the market and, thereby, reduce price competition between firms. The reason is that copying by low, more pricesensitive types enables firms to credibly charge higher prices on the segment of consumers that do not copy. Furthermore, this positive effect of piracy on firms' profits can sometimes outweigh the negative impact due to lost sales. So, even in the absence of network effects, firms may prefer weak copyright protection in equilibrium.

Finally, there is a recent paper by Minnitti and Vergari (2010), who also rely on the Hotelling differentiated-product duopoly framework. They, however, deal with a rather specific form of piracy like a private file sharing community and study how its presence affects the pricing behavior and profitability of producers of digital products.

It is also important to note that digital developers' competition can also occur in a multiproduct framework, where piracy can generate a kind of indirect competition between horizontally differentiated digital products as demonstrated by Belleflamme and Picard (2007). They show how the copying technology displaying increasing returns to scale can create an interdependence between the demands for digital products that would be unrelated otherwise. Moreover, the underlying demand is, much like in our approach, obtained in a vertical differentiation manner. However, the vertical differentiation does not, like in our set-up, arise from the different quality levels of the developers but from the existence of original and copied digital products in a market where the originals are assumed to be always of higher quality than the copies, and thus, all consumers unambiguously prefer the original product over the copy. In this set-up Belleflamme and Picard (2007) study how piracy affects prices and profits and, interestingly enough, they show that depending on the parameters of the model, prices can be either strategic substitutes or strategic complements. If the fixed cost of copying is low enough, there is no equilibrium in pure strategies. Firms may then randomize between several prices, leading to price dispersion.

Following the approach of Belleflamme and Picard (2007), Choi, Bae, and Jun (2010) use 
a Hotelling horizontal differentiation model as well and analyze the situation in which also the interdependence between the firms stems from their strategies against piracy rather than from direct competition on prices. They, like we do, consider the IPR efforts of the firms to be endogenous variables and study the interaction between public and private protection against piracy.

Besides the different focus (direct versus indirect competition), the other key difference between ours and the set-up of Belleflamme and Picard (2007) and Choi, Bae, and Jun (2010) is that in their settings the original products have the same quality, while in our set-up, the original products are vertically differentiated and thus have distinct qualities to begin with. Moreover, since we focus on the software market, we do not allow for a different copying technology as it is usual in the case with multiple, initially independent digital products. Thus, the cost of consuming illegal copies is constant in our setting, while it may be decreasing with the number of different originals copied in the settings of Belleflamme and Picard (2007) and Choi, Bae, and Jun (2010).

Finally, there are by now numerous scholarly articles that deal with the issue of digital piracy and private or public IPR protection in the monopoly set-up (see, for instance, Banerjee, 2003; King and Lampe, 2003; Kúnin, 2004; Takeyama, 2009). Banerjee (2003) demonstrates that the socially optimal level of IPR protection differs from a monopoly developer's optimum and stresses the role of NEs. King and Lampe (2003) show that the monopoly allows illegal users in the case when the network effect is present, while Takeyama (2009) shows that under asymmetric information about product quality, the copyright has to be imperfect in order to avoid adverse selection. Kúnin (2004) provides an explanation for why a software manufacturer may tolerate widespread copyright infringement in developing countries and often even offer local versions of their software. He showed that if NEs are present and there is an expected improvement in copyright, then software manufacturers enter the market even if they incur losses in the beginning when copyright enforcement is weak. For a deeper and systematic review of the literature on the piracy of digital products, the interested reader is advised to look at the two excellent and comprehensive surveys in Peitz and Waelbroeck (2006) and Belleflamme and Peitz (2011).

As already mentioned above, we focus our analysis on the developers' strategic interactions and the way how the size of the expected penalty affects market structure, market coverage, and the developers' IPR protection. We especially put the emphasis on the latter, meaning on the interaction between the government's (or public) and the developer's (or 
private) IPR protection. We show that when developers restrict services to illegal users (section 3), the government's and the developers' IPR are always substitutes in a sense that for the given developers' optimal protection, the public IPR protection could be substantially lower (compared to the situation with no private IPR protection) in order to fully eliminate illegal usage. Moreover, the government can by choice of its IPR protection (that is, via the size of the expected penalty) affect the market configuration and market coverage since the height of the expected penalty has an effect on equilibrium prices and profits and thus on the toughness of price competition. For instance, for the size of the expected penalty that falls between two prices, there might occur a market configuration with two unconnected segments of legal users. In this case, the high quality developer serves the upper part of the market and earns (constrained) monopoly profit, while the lower quality developer serves the lower tail of the market. In the middle of these two segments, there is a "buffer" composed of illegal users ${ }^{18}$. If on the other hand, the government sets penalty rather low so that both prices $^{19}$ are bigger than the expected penalty, then direct duopoly competition might be restored.

As for the situation when developers rely on the physical protection of their software (section 4), the government's and the developers' IPR could be either substitutes or complements in a sense that a marginal increase in the expected penalty can either decrease the optimal developer protection (implying substitutes) or increase it (implying complements). Moreover, the size of the expected penalty is a key in determining whether none, one, or both developers would introduce private IPR protection. If the expected penalty is so low that both prices exceed it, then both developers would introduce protection, and a small increase in the expected penalty would reinforce the developer protection indicating the complementarity of the two forms of protection. If on the other hand, the expected penalty exceeds the price of the low quality good but is still lower than the price of the high quality good, then only the high quality developer would introduce IPR protection. Now, however, a marginal increase in the expected penalty would decrease the optimal developer protection implying substitutability between the two forms of protection.

The structure of the essay is the following: In the second chapter, we put forward our setup that comprises three basic types of market conduct: monopoly, Bertrand duopoly, and the

\footnotetext{
${ }^{18} \mathrm{~A}$ necessary condition for this case to arise is that an illegal copy of a high quality product has a higher value for users than the quality of a legal, lower quality product.

${ }^{19}$ Meaning equilibrium prices in standard Bertrand competition, where illegal usage is eliminated.
} 
Stackelberg leader-follower model. We then analyze how the level of EP affects the developers conduct and market structure in the simplest case when there is no product protection from the developers' side whatsoever. In the third chapter, we allow the developers to introduce product protection in the form of a lower product value for illegal users by disabling them access to additional services. In the fourth chapter, we investigate the economic impacts of another form of product protection in which a developer implements a physical protection for his product. In both the third and fourth chapters, we study the effects of the particular form of developer's IPR protection within the three above mentioned market conducts. Chapter five concludes.

\section{The basic model}

We first analyze the cases where developers could eliminate the illegal usage of their products only by decreasing prices. Developers cannot introduce any product protection or restrict associated product services to the illegal users.

\subsection{Model set-up}

\subsubsection{Industry set-up}

Consider two developers $A$ and $B$ that compete in prices on a particular market and offer product varieties of different quality ${ }^{20}$. Developer $A$ releases a product of quality $q_{A}$, while the quality of the second developer $B$ is $q_{B}$ and we assume, without loss of generality, in the rest of the essay that developer $A$ offers higher quality $\left(q_{A}>q_{B}\right)$. Product qualities $q_{A}, q_{B}$, in the whole essay are assumed to be exogenous and cannot be changed by developers ${ }^{21}$ The unit variable costs are assumed to be constant and normalized to zero. One may think about developer $A$ as an already established and known software producer that already operates on other markets. This fact is, in turn, reflected in the preferences of the consumers, who strictly prefer software $A$ over software $B$ if offered at the same price. Similarly, developer

\footnotetext{
${ }^{20}$ We will use the term "value" instead of "quality" when quality contains multiple dimensions.

${ }^{21}$ In the more elaborated versions of this kind of models, there is also a choice of qualities proceeding the pricing decision. In this case, it is standard to assume that the bulk of the costs of generating quality falls on fixed costs so that quality or R\&D costs are in fact endogenously determined (see, for instance, Shaked and Sutton, 1982 and 1983; Kúnin and Žigić, 2006). For each case that we analyze, it should be clear how to relax the model and allow the developers to choose and compete in qualities too.
} 
$B$ can be thought of as a local developer offering lower quality. In other words, we assume that both developers already existed before meeting and competing on the market under consideration. Consequently, both developers are assumed to have already incurred setup fixed costs and fixed costs associated with software development (R\&D costs). These fixed costs are, from our perspective, general and not related to the developer's presence on the particular market under consideration, and therefore, we leave them out of the profit function. We, however, may allow for the fixed costs of entry to the particular market under consideration, so we denote as $F_{A}$ and $F_{B}$ these entry or set-up costs respectively (sinking these costs can be considered to take place at the first stage of the game). We will, however, omit these fixed costs from the profit functions for the purpose of transparency and assume that the developers' profits are positive net of these costs.

To summarize, we simply assume that:

1. Initially, both developers $A$ and $B$ already exist with established quality levels of their respective varieties.

2. The focus is on a particular software market, which is not interrelated with the other markets on which developers may operate ("segmented market hypothesis").

Thus, it is convenient to think that two developers compete (or may compete) in some third market (that is, the market that is not their home market). An important implication of these two assumptions is that in our set-up one or even both developers may not be active in the market under the considerations. The reason for this is that due to the absence of the developers' own IPR protection and the possible lack of IPR protection by the side of the regulator, it may not be profitable for the developer(s) to operate in the market under considerations. We, however, assume that even if a developer does not enter the market, the users are still able to obtain an illegal version via copying. This, in turn, makes entry deterrence not viable. We use a sub-game perfect equilibrium as a solution concept throughout this paper in all multi-period games under considerations.

\subsubsection{The regulator's role}

We introduce a very simple regulator whose role is limited to monitoring software usage and to the penalization of those users, who use products illegally and are disclosed. The probability of being caught using an illegal version is the same for all users, and the level of the 
penalty is fixed. The penalty and the probability of being caught is known and independent on used product and product prices ${ }^{22}$, thus all users and both developers could calculate the expected penalty for using an illegal version, that we denote as $X^{23}$. Moreover, while we implicitly assume that the regulator choice of optimal IPR is governed by an underlying objective function like the maximization of social welfare, we do not explicitly study the optimal choice of EP since we focus on the forms of the developers' IPR protection and their economic implications ${ }^{24}$. Thus, the whole regulator's framework ${ }^{25}$ is very simple in our model and translates into one parameter: expected penalty $X$ for illegal users.

\subsubsection{The users' set-up}

There is a continuum of risk neutral heterogenous users on a particular market under the considerations that differ in their personal value of product quality $q_{i}$, captured by parameter $\theta$, where $\theta$ follows a uniform distribution over the interval $(0, \bar{\theta})$. Following Tirole (1988), utility for a user $\theta$ from consuming product $q_{i}$ with price $p_{i}$ and with expected penalty $X$ from illegal usage is the following ${ }^{26}$ :

$$
U_{\theta}\left(p_{i}, q_{i}, X\right)=\left\{\begin{array}{lll}
\theta q_{i}-p_{i} & \ldots & \text { if a user buys software } \\
\theta q_{i}-X & \ldots & \text { if a user uses an illegal version } \\
0 & \ldots & \text { if a user does not use the software at all }
\end{array}\right.
$$

From the users' utility function, we immediately see that for $p_{i} \neq X$, there are two groups of indifferent users. The users, who are indifferent between buying a product and not using it at all $\left(\theta q_{i}-p_{i}=0\right)$, denote them as $\theta_{0 A}=\frac{p_{A}}{q_{A}}$ (respectively $\left.\theta_{0 B}=\frac{p_{B}}{q_{B}}\right)$, and users who

\footnotetext{
${ }^{22}$ The penalty in the real economy is dependent on the price of software as well as the social impact from such behavior and real losses.

${ }^{23} \mathrm{We}$ can expect that in a real economy, the fines for students compared to business companies differs as well as the regulator's monitoring rate among students/households is lower than in business. The extension of the model for different $X$ for each product (different users) would lead to technical complication, that from our focus would add only limited further insight.

${ }^{24}$ For instance, if the government maximizes social welfare, we would need to know which of the developers is the domestic one and which is not in order to write down the objective functions. While these considerations are interesting per se, they are not the focus of the essay. For the analysis of the optimal IPR from the side of the regulator, see for instance Žigić (2000).

${ }^{25}$ Including government behavior, legal environment, and the real execution of property rights.

${ }^{26}$ To avoid the problem of a fully satiated market, we do not follow distribution over $\left(\theta_{l}, \theta_{h}\right)$ as in Banerjee (2003). Allowing the presence of users with $\theta \rightarrow 0$ ensures that for any price $p>0$, there is a group of users who do not consume any product.
} 
are indifferent between illegal usage and not using it at all $\left(\theta q_{i}-X=0\right)$, denote them as $\theta_{0 P}=\frac{X}{q_{i}}$.

\subsection{Monopoly}

We start with the simplest set-up, where only developer $A$ with a product of quality $q_{A}$ is present on the market, and there is perfect IPR enforcement. This set-up means that the expected penalty $X$ is higher than monopoly the equilibrium price for the legal product $p_{M}^{*}$ (2), and thus, nobody uses the product illegally. Developer $A$ maximizes profit without any restriction, and from utility function (1), we see that $\theta_{0 A}=\frac{p_{M}}{q_{A}}$ is the user indifferent between buying software and not using it at all. That leads to the following demand for product $A$ :

$$
D_{M}\left(p_{M}, q_{A}\right)=\bar{\theta}-\theta_{0 A}=\bar{\theta}-\frac{p_{M}}{q_{A}},
$$

so that the monopoly equilibrium price $p_{M}^{*}$ and profit $\pi_{M}^{*}=p_{M}^{*} D_{M}\left(p_{M}^{*}, q_{A}\right)$ are:

$$
\pi_{M}^{*}=\frac{1}{4} q_{A} \bar{\theta}^{2}, p_{M}^{*}=\frac{1}{2} \bar{\theta} q_{A}
$$

With such a price, developer $A$ captures half of the market, $\frac{1}{2} \bar{\theta}$ (see Figure 1 ).

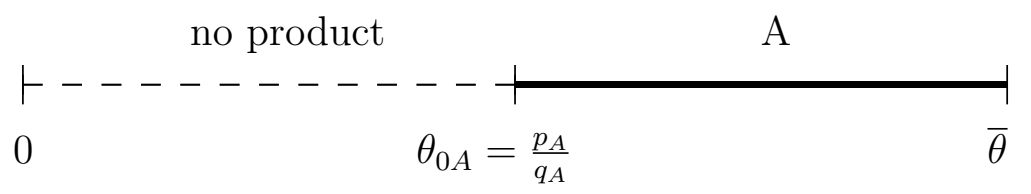

Figure 1: Monopoly market

Now for a while, assume that developer $A$ operates on the monopoly market where IPR enforcement is lower than would be desirable for him, that is, $X<p_{M}^{*}=\frac{1}{2} \bar{\theta} q_{A}$. In this case, users compare the expected penalty $X$ with price $p_{M}$, so all users with $\theta \geq \frac{X}{q_{A}}$ prefer illegal software usage. The only possibility for developer $A$ to capture some legal users on the market is to lower the price to the level $p_{M}^{*}=X$. This is the case when developer $A$ has to adjust the price to the level "set" by the regulator (that is, to the the level of $X$ ); otherwise, the developer is out of the market. Under such a situation, the regulator could effectively influence the developer's price $p_{M}$, which results in the following profit for developer $A$ :

$$
\pi_{M}^{*}=\left(\bar{\theta}-\frac{X}{q_{A}}\right) X
$$


and the market share that the developer gains is $\left(\bar{\theta}-\frac{X}{q_{A}}\right)$, which is higher than $\frac{1}{2} \bar{\theta}$ from equilibrium (2).

\subsection{Bertrand Competition}

After the analysis of the basic case with a monopoly developer, consider now Bertrand competition in prices between developers $A$ and $B$. In this case, low $X$ might not be a constraint only for developer $A$ but even for developer $B$. Thus, three basic cases of competition exist based on the presence of developer $A$ in the market and on the level of the expected penalty $X$ with respect to equilibrium prices $p_{A}^{o}, p_{B}^{o}$, where these prices come from pure Bertrand competition in ( 4 ):

1. $p_{A}^{o}, p_{B}^{o} \leq X \quad \ldots$ High $X$ : none of developers are limited in price setting by the level of $X$.

2. $\frac{X}{q_{A}} \leq \frac{p_{B}^{o}}{q_{B}} \leq \frac{p_{A}^{o}}{q_{A}} \quad \ldots \quad$ Low $X$ : both developers are limited in price setting by the level of $X$

3. $\frac{p_{B}^{o}}{q_{B}} \leq \frac{X}{q_{A}} \leq \frac{p_{A}^{o}}{q_{A}} \quad \ldots \quad$ Medium $X$ : only developer $A$ is limited in price setting by the level of $X$

Notation 1 In indexing indifferent users, $P$ will always refer to illegal usage, 0 to not using any product at all, and $A, B$ always refer to using (legally) the product $A, B$ respectively. Moreover, we follow the rule that the first index refers to a user "on the left-hand side" and the second index will refer to a user "on the right-hand side." For instance, $\theta_{P B}$ means that a user with a lower $\theta$ than $\theta_{P B}$ uses the product illegally, while a user with $\theta$ higher than $\theta_{B P}$ legally uses product $B$. As for $\theta_{B P}$, the same applies the other way around.

\subsubsection{Case 1: Bertrand competition under a high expected penalty $p_{A}^{o}, p_{B}^{o} \leq X$}

This first basic case, where all piracy is eliminated, corresponds to the pure Bertrand competition $^{27}$, where both developers could freely compete in prices. Denote a user who is indifferent between product $A$ and $B$ as $\theta_{B A}=\frac{p_{A}-p_{B}}{q_{A}-q_{B}}$. Then profit functions for both developers are:

$$
\pi_{A}=\left(\bar{\theta}-\theta_{B A}\right) p_{A}, \pi_{B}=\left(\theta_{B A}-\theta_{0 B}\right) p_{B}
$$

\footnotetext{
${ }^{27}$ It might be quite an unrealistic case that does not mimic an Operating System or Office Packages submarkets (or other, on retail focussed, markets), nevertheless specific business software markets, e.g. CAD systems, are close to such situation. In such cases, the illegal usage of software often precedes official buying and exists mainly because of testing purposes.
} 
This situation corresponds to the market coverage as in Figure 2 .

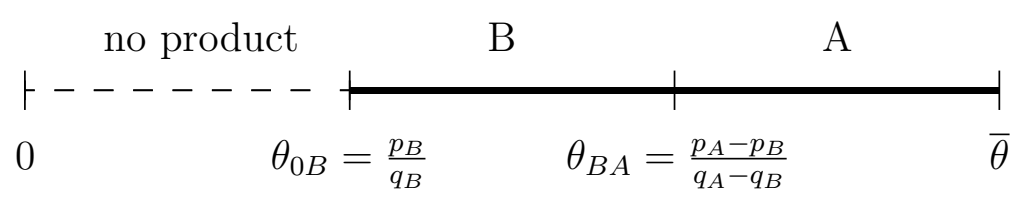

Figure 2: Standard Bertrand Competition

From F.O.C. and S.O.C., stated in Appendix 1.3.1, we obtain equilibrium prices and profits for both developers:

$$
\begin{gathered}
p_{A}^{o}=2 \bar{\theta} q_{A} \frac{q_{A}-q_{B}}{4 q_{A}-q_{B}}, p_{B}^{o}=\bar{\theta} q_{B} \frac{q_{A}-q_{B}}{4 q_{A}-q_{B}} . \\
\pi_{A}^{o}=4 \bar{\theta}^{2} q_{A}^{2} \frac{q_{A}-q_{B}}{\left(4 q_{A}-q_{B}\right)^{2}}, \pi_{B}^{o}=\bar{\theta}^{2} q_{B} q_{A} \frac{q_{A}-q_{B}}{\left(4 q_{A}-q_{B}\right)^{2}} .
\end{gathered}
$$

Corollary 1 Equilibrium with $p_{A}^{o}, p_{B}^{o} \leq X$ exists if and only if $X \geq 2 \bar{\theta} q_{A} \frac{q_{A}-q_{B}}{4 q_{A}-q_{B}}$. This could be immediately seen from (4).

Obviously, both the prices and the profits of each developer increases when value of his own product increases or when a competitor's product value decreases. We see directly from the equilibrium that the relationships between prices and profits are the following:

$$
\frac{p_{A}^{o}}{p_{B}^{o}}=2 \frac{q_{A}}{q_{B}}, \frac{\pi_{A}^{o}}{\pi_{B}^{o}}=4 \frac{q_{A}}{q_{B}} .
$$

We refer to this case as the pure Bertrand competition.

\subsubsection{Case 2: Bertrand competition under low expected penalty: $\frac{X}{q_{A}} \leq \frac{p_{B}^{o}}{q_{B}} \leq \frac{p_{A}^{o}}{q_{A}}$}

Now we focus on the second case when the expected penalty $X$ is lower than the level that would allow for the pure Bertrand competition stated above (which means $\frac{X}{q_{A}} \leq \frac{p_{B}^{o}}{q_{B}} \leq \frac{p_{A}^{o}}{q_{A}}$ ). We assume here that developer $A$ cannot decrease the price at the level of (or below) $X$ due to large entry costs, for instance, and developer $B$ cannot react on it as well. (The case when both developers could adjust the price accordingly to $X$ is analyzed as a special case in the next sub-section-Case 3). Putting $p_{B}^{o}$ from (4) into $\frac{X}{q_{A}} \leq \frac{p_{B}^{o}}{q_{B}}$, we see immediately that $X$ must be lower than $\bar{\theta} q_{A} \frac{q_{A}-q_{B}}{4 q_{A}-q_{B}}$. In this case, the expected penalty is so low that all users prefer to use the product illegally. Since the expected penalty $X$ is the same no matter which product is used, all users illegally use the product of higher quality $q_{A}$ from developer A. It corresponds to the market coverage as in Figure 3 . 


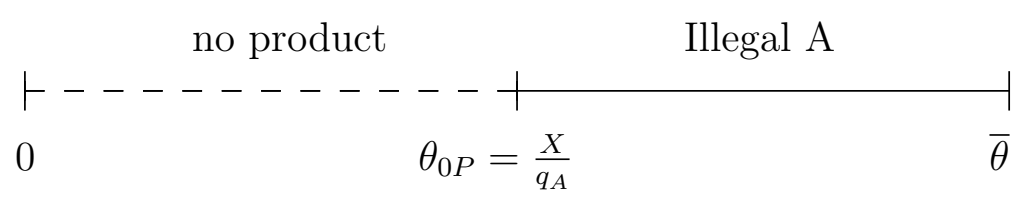

Figure 3: No legal version on the market

Remark 1 Though this situation might seem implausible because none of the developers may generate profit unless they set their prices such that $p=X$, we could, nevertheless, find such a situation at which IPR practically does not exist ( $X$ goes to zero), and a price reduction from the developers would not lead to significantly higher sales ${ }^{28}$. At such markets, developers officially do not operate and are active on other markets, so we observe only the demand side of the market. Alternatively in dynamic models, developers may operate in those markets anticipating an improvement in IPR and so expecting to achieve profit in the future based on the established market position today, see Kúnin (2000).

\subsubsection{Case 3: Bertrand competition under medium expected penalty $\frac{p_{B}^{o}}{q_{B}} \leq \frac{X}{q_{A}} \leq$ $\frac{p_{A}^{o}}{q_{A}}$}

In this case, we assume that the expected penalty $X$ influences only developer $A$ since his equilibrium price $p_{A}^{o}$ is higher than $X$ (while still we have $p_{B}^{o}<X$ ). From (4), we see that $\bar{\theta} q_{B} \frac{q_{A}-q_{B}}{4 q_{A}-q_{B}} \leq X$, and we assume for the moment that developer $A$ is not present in the market due to, say, large set-up costs that exceed profit. Then there are users who prefer to buy product $B$ rather than face the risk of being caught as a user of an illegal version, while potential users of product $A$ prefer to use an illegal version of product $A$. In such a market, developer $B$ competes with an illegal version of software $A$ in a sense that the upper part of the market that belonged to developer $A$, as in Case 1 , is now occupied by the illegal users of $A$. A user $\theta_{0 B}$ who is indifferent between using product $A$ illegally and product $B$ legally satisfies $\theta_{B P} q_{A}-X=\theta_{B P} q_{B}-p_{B}$. A user indifferent between using product $B$ legally and not using any product at all satisfies $\theta_{0 B} q_{B}-p_{B}=0$. Thus, $\theta_{B P}=\frac{X-p_{B}}{q_{A}-q_{B}}$ and $\theta_{0 B}=\frac{p_{B}}{q_{B}}$. This market situation leads to product coverage across users as in Figure 4 .

Developer $B$ captures market share $\theta_{P B}-\theta_{0 B}$, while an illegal version of product $A$ is

\footnotetext{
${ }^{28}$ Those are developing/emerging markets with very low IPR protection, where the percentage of illegal versions can be higher than 95\% - e.g., the illegal usage evaluated in Vietnam according to the BSA (Business Software Alliance).
} 


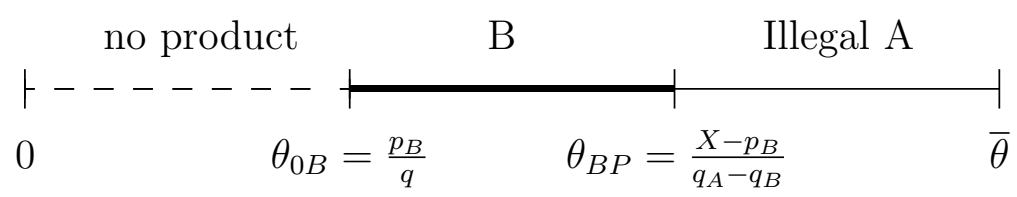

Figure 4: Only developer $B$ is on the market with a legal version

used by $\bar{\theta}-\theta_{B P}$ users. In this case, only developer $B$ makes some profit $\pi_{B}=\left(\theta_{B P}-\theta_{0 B}\right) p_{B}$. Equilibrium profits and prices are derived in Appendix 1.3.2 :

$$
p_{B}^{*}=\frac{q_{B}}{2 q_{A}} X, \pi_{B}^{*}=\frac{q_{B}}{4 q_{A}} \frac{X^{2}}{\left(q_{A}-q_{B}\right)} .
$$

Developer $A$ could decrease his price to a level $p_{A}=X$, and this behavior does not affect market share or the profit of developer $B$ (see subcase 2.3.4 and Figure 5 ).

In real life, this market situation corresponds to the competition between a small local developer producing a lower quality product with a global developer, who may even not formally operate on the market. Close to this market situation, was the situation with system utilities and antivirus programs in Russia or in China around the year 2000 when global developers were waiting for improvement in IPR protection and making very negligible profit.

In this case where $\frac{p_{B}^{o}}{q_{B}} \leq \frac{X}{q_{A}} \leq \frac{p_{A}^{o}}{q_{A}}$, developer $A$ could decrease the price to level $p_{A}=X$ and compete with developer $B$ with this adjusted price, so we focus next on this interesting subcase.

\subsubsection{Bertrand competition with the binding price $p_{A}^{*}=X$}

Note that in Cases 2 and 3 where $X$ is low, developer $A$ has the possibility to decrease the price to $p_{A}^{*}=X$. The costs of this is that developer $A$ has to incur the set-up costs $F_{A}$. Comparing it with Case 1, that price adjustment puts pressure on developer $B$ to also lower his price $p_{B}$, so it results in a decreased profit for both developers. This market situation leads to the following equilibrium prices and profits (see Appendix 1.3.3 ):

$$
\begin{aligned}
& p_{A}^{*}=X, \quad p_{B}^{*}=\frac{1}{2} \frac{q_{B}}{q_{A}} X . \\
& \pi_{A}^{*}=\frac{1}{2} X \frac{\left(X\left(q_{B}-2 q_{A}\right)+2 \bar{\theta} q_{A}\left(q_{A}-q_{B}\right)\right)}{q_{A}\left(q_{A}-q_{B}\right)}, \pi_{B}^{*}=\frac{q_{B}}{4 q_{A}} \frac{X^{2}}{\left(q_{A}-q_{B}\right)} .
\end{aligned}
$$

Nevertheless, this price adjustment forced by a lower $X$ leads to a similar market distribution as in the first case, where $X \geq p_{A}^{*}, p_{B}^{*}$, but the total number of users is now higher. Market 
coverage is displayed in figure 5 .

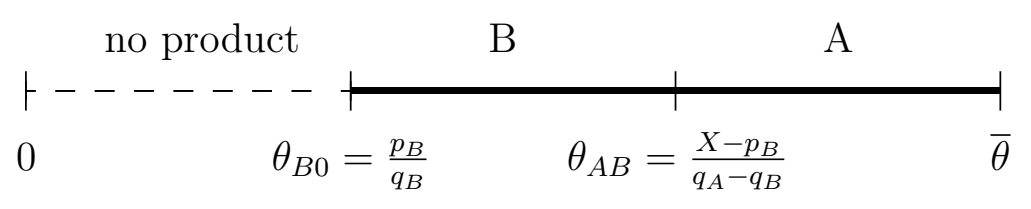

Figure 5: Competition with the adjusted price $p_{A}$

Lemma 1 Relative prices are proportional to the corresponding quality levels and are the same as the ratio of the price derivatives with respect to $X$. As for the respective profits, note that the profit for developer $A$ increases by a larger amount in $X$ than the profit for developer $B$ :

$$
\begin{aligned}
\frac{p_{A}^{*}}{p_{B}^{*}} & =\frac{\frac{\partial p_{A}^{*}}{\partial X}}{\frac{\partial p_{B}^{*}}{\partial X}}=2 \frac{q_{A}}{q_{B}}, \\
\frac{\partial \pi_{A}^{*}}{\partial X} & =\frac{2 \bar{\theta}}{X} \frac{q_{A}\left(q_{A}-q_{B}\right)}{q_{B}}-2\left(2 \frac{q_{A}}{q_{B}}-1\right) .
\end{aligned}
$$

Obviously an increase in $X$ implies an increase in $p_{A}$ by the same amount (that is $\frac{\partial p_{A}(X)}{\partial X}=$ 1 ), while the induced rise in $p_{B}$ is much lower $\left(\frac{\partial p_{A}(X)}{\partial X}=\frac{1}{2} \frac{q_{B}}{q_{A}}<\frac{1}{2}\right)$. Consequently, an increase in developer $A$ 's profit (due to a rise in $X$ ) is larger than the increase in developer $B$ 's profit despite the fact that market coverage of developer $A$ shrinks at the expense of developer $B$. Note that developer $B$ gains the lower tail of developer $A$ 's market, and this gain exceeds the loss of the lower tail of his own market. The latter occurs due to an increase in $p_{B}$ induced by an increase in $X$. In other words, $\frac{\partial}{\partial X}\left(\theta_{B A}-\theta_{0 B}\right)=\frac{1}{2\left(q_{A}-q_{B}\right)}>0$.

\subsection{Stackelberg competition in prices}

In this part, we focus on Stackelberg (Leader-Follower) competition and particularly only on the situation when both developers are present on the market:

1. $X>p_{A}^{*}, p_{B}^{*} \quad \ldots$ high expected penalty $X$, that no developer is restricted by $X$.

2. $p_{A}=X \quad \ldots \quad$ Developer $A$ is limited in price setting by the expected penalty.

The other cases are equivalent to the cases analyzed in Bertrand competition and monopoly set-up in the previous part. Note, that in our analysis of the Stackelberg framework, we always assume that developer $A$, who releases a higher quality product $q_{A}$, is a price leader, while developer $B$ is a price follower ${ }^{29}$.

\footnotetext{
${ }^{29}$ Assuming that developer $B$ is the price leader could be possible for selected sub-markets that correspond
} 


\subsubsection{Stackelberg competition with a high expected penalty $p_{A}^{*}, p_{B}^{*} \leq X$}

Since developer $A$ is the price leader who knows the reaction function of developer $B$, he incorporates this reaction function of developer $B$ (that is $p_{B}\left(p_{A}\right)=p_{A} \frac{q_{B}}{2 q_{A}}$ ) into his profit function, and thus we obtain equilibrium prices and profits:

$$
\begin{aligned}
p_{A}^{*} & =\frac{q_{A}-q_{B}}{2 q_{A}-q_{B}} q_{A} \bar{\theta}, p_{B}^{*}=\frac{1}{2} \frac{q_{A}-q_{B}}{2 q_{A}-q_{B}} q_{B} \bar{\theta}, \\
\pi_{A}^{*} & =\frac{1}{2} \frac{q_{A}-q_{B}}{2 q_{A}-q_{B}} q_{A} \bar{\theta}^{2}, \pi_{B}^{*}=\frac{1}{4} \frac{q_{A}-q_{B}}{\left(2 q_{A}-q_{B}\right)^{2}} q_{B} q_{A} \bar{\theta}^{2} .
\end{aligned}
$$

(For F.O.C. and S.O.C., see Appendix 1.4.1 .)

Remark 2 Compared with Bertrand competition, the profit of developer $B$ is always higher in Stackelberg competition.

In the next section, we will show a case (a buffer case), where developer $B$ is indifferent between Stackelberg and Bertrand competitions since both frameworks bring him exactly the same profit.

\subsubsection{Stackelberg competition with binding price $p_{A}^{*}=X$}

Assume a market situation with a low expected penalty $X$ which becomes binding (if $X \leq$ $\left.\frac{q_{A}-q_{B}}{2 q_{A}-q_{B}} q_{A} \bar{\theta}\right)$. Then, equilibrium prices and profits are the same as in the case of Bertrand competition with binding $X$ as stated in 2.3 .4 :

$$
\begin{aligned}
& p_{A}^{*}=X, p_{B}^{*}=X \frac{q_{B}}{2 q_{A}}, \\
& \pi_{A}^{*}=\frac{1}{2} \frac{\left(2 \bar{\theta} q_{A}\left(q_{A}-2 \bar{\theta} q_{B}\right)-X\left(q_{A}-q_{B}\right)\right) X}{q_{A}\left(q_{A}-q_{B}\right)}, \pi_{B}^{*}=\frac{1}{4} \frac{q_{B}}{q_{A}} \frac{X^{2}}{q_{A}-q_{B}} .
\end{aligned}
$$

(For a derivation see Appendix 1.4.1 .) In the Stackelberg case, the distribution of users on the market is equal to the Bertrand competition. The market coverage is the same as in Figure 5 .

to the situation, where $B$ is well established main stream player, while $A$ is a niche player for a small proportion of high-end users, and $A$ has to adjust his price according to the main stream. However, analyzing this market structure would not add value to this essay. 


\subsection{Possible Network Effect Extension}

Before concluding this section and approaching an analysis of product protection, we make small remarks on Network Effect since NE plays an important role on some software submarkets. In these remarks, we will show a possible incorporation of the Network effect into the model.

Capturing the significant base of users may allow a developer to extract additional value from those users. In such a situation, the developer may tend to predatory behavior (entering the market with a low price to capture base, and increasing the price later), when he deliberately supports illegal copying to raise the user base and thus user value from a product and in the future, implement protection which results in "locking" the base of users (see Farrell and Klemperer, 2006 $)^{30}$. In non-predatory competition, the NE could raise the product's value substantially. Our set-up would allow for the capturing of the NE by internalizing it into the product quality. Assume now that $q_{i}$ is composed from:

$$
q_{i}=\beta_{A} \cdot Q_{i}+\left(1-\beta_{A}\right) N E_{i},
$$

where $Q_{i}$ is the quality of product itself, and $N E_{i}$ is the value that a user puts into the user base, and $1 \geq \beta_{A} \geq 0$ is the weight of each component. In general, using the model with the NE we would search for the optimal value of each component $Q_{i}$, and $N E_{i}$, and the equilibrium would consist of $p_{i}^{*}, Q_{i}^{*}$, and $N E_{i}^{*}$. Such an equilibrium would be strongly dependent on weights $\beta_{A}$ and the set-up cost functions $F_{A}\left(Q_{A}\right), F_{B}\left(Q_{B}\right)$. Given the main focus of the essay, an extension of the set-up for the NE would not bring significant additional insights to our analysis.

Moreover, the NE starts to become a less important driving factor for most software submarkets recently, and especially, the NE is even already quite suppressed for industrial software. The more important factor becomes software compatibility among competitors and their mutual replacement ability (e.g. functions or layout) ${ }^{31}$.

\footnotetext{
${ }^{30}$ Usually solved by a two-period model, where in the first period a developer raises his user base and in the second period, charges them for the additional value from the user base.

${ }^{31}$ Thus, in the case of analyzing the Network Effects, we should always distinguish cases where products $A$ and $B$ are mutually incompatible, partially compatible, or fully compatible. The compatibility is then a factor that allows the competitors to exploit the user base and to suppress the NE advantage of a particular product.
} 


\subsection{Key chapter results}

From the analysis in Section 2, we see that imposing penalties has a strong impact on the resulting market coverage. In the case of the high expected penalty $X$, there is standard competition in prices (either Bertrand or Stackelberg), while in the case of the medium expected penalty $X$, the developer with the higher quality product has to either leave the market or decrease the price. In such a market situation, $X$ fosters the competition and forces both developers to decrease prices, but at the same time, too low of an $X$ could squeeze one of the developers out of the market since he may not be able to recover his set-up costs any longer. Thus, a very low $X$ increases the toughness of price competition that in turn may result in a monopoly market structure. From the government's point of view, $X$ may serve as an artificial price, set by the regulator, that must either be accepted by developer $A$, or he has to leave the market. In the case of a very low $X$, none of the developers would operate on the market.

\section{Decreasing product value for illegal users}

In the previous section, users did not perceive a quality (value) difference between the original product and its illegal version, and thus, users always chose the version with a lower "cost" per quality unit $\left(\frac{p_{i}}{q_{i}}\right.$ in the case of a legal version and $\frac{X}{q_{i}}$ in the case of an illegal version). In this chapter, we assume that the legal and illegal versions are no more perfect substitutes. That is, the value of the legal version differs from the illegal version since a developer provides part of valuable services only to legal users (such as online help and technical support, live updates, a discount for upgrades or even free training, access to user manuals, etc.). Probably the most famous example of restricting services to illegal users, familiar to everybody, is the one with Microsoft Windows. Microsoft's Windows Genuine Program allows a user to run an illegal version of the product only up to a certain point. In order to install selected patches/updates, the user has to validate the originality of the program online. If a particular copy is identified as illegal, some functions are disabled, and the illegal user is irritated with constant messages about buying the legal version. If a user decides not to validate the program online, he cannot update his Windows further for selected components (e.g. Windows Media Player or Internet Explorer $)^{32}$. The implementation of such a restriction

\footnotetext{
${ }^{32}$ Another examples is antivirus programs (e.g., Symantec Antivirus), when often after updates, the program recognizes that a particular installation is illegal and does not allow further updates of its virus database
} 
is technically easy since the developer could use the standard tools that restrain access to those services $^{33}$ that require user authorization based on personal information verification. In the case of automatic access to those services, a developer can use very reliable tools as authorization is based on the IP address or hard-locks.

\subsection{Model set-up}

\subsubsection{Industry set-up}

We now assume that developers cannot directly restrict illegal usage of the product itself but could restrict part of the services related to the product. This restriction lowers the product value for illegal users. Denote the value (perceived quality) of the legal version as $q_{i}$. The exclusive part of the product value that only the legal users can enjoy is $1-\alpha$, where $\alpha \in(\underline{\alpha}, 1)$, and $\underline{\alpha}>0$ stands for technically the lowest possible level of restriction beyond which it is impossible to further restrain services ${ }^{34}$. Thus, the value of the product for the illegal users is decreased ${ }^{35}$ to $\alpha q_{i}$. As for the developers' costs of restricting services, it seems reasonable to assume that these costs are negligible given that the developers already exist and have chosen their quality levels and the accompanied level of consumer services previously. So, we assume these costs to be zero, but we do discuss the implications of nonzero costs for the optimal choice of $\alpha$ in section 3.4. For simplicity, we assume that if both developers choose to restrict services, then they would choose the same $\alpha^{36}$. In a formal sense, adding the possibility for the developer to choose the degree of service restrictions can be considered as a two-stage game: In the first stage, one or both developers choose the degree of service restrictions $\alpha$, and in the second stage, the developers compete in prices.

for new viruses. Finally, for many computer games, online playing is allowed only for the legal users.

${ }^{33} \mathrm{~A}$ separate question is whether a developer could provide enough services/online content that would bring a user enough additional value to offset the difficulties with accessing those services/content.

${ }^{34}$ Technically, the lowest possible $\underline{\alpha}$ represents such $\alpha$ where quality $q_{A}$ to legal users is not affected. By decreasing $\alpha$ below $\underline{\alpha}$, we assume that it would require such strong protection/verification tools (e.g. manual online authorization), which become annoying even for legal users, and their value assigned to product $q_{A}$ would drop.

${ }^{35}$ Banarjee (2003) explains the difference between an original and illegal version as the probability of an occurrence of a defect illegal version. In his framework, "not" defected illegal copy is the same for a user as a legal copy.

${ }^{36}$ Allowing for different $\alpha$ would in no way change qualitatively our analysis. It would make the results and analysis only less transparent since it would always require a comparison between illegal version values, see Bertrand competition in 3.3 . 
In what follows, we focus on the second stage of the game in which a developer chooses the prices and analyze the impact of different $\alpha$ on equilibrium prices and on the resulting market structure and coverage. In the last sub-section of this chapter, we briefly discuss the optimal choice of service restrictions.

Note that in the case of a low expected penalty, $X$, that results in a "protecting" action from the developer, we could perceive $X$ as public protection while an action from a developer (in this case introducing $\alpha$ ) as private protection.

Remark 3 Note that developer $B$ has somewhat limited incentives to restrain certain services to illegal users. Since the expected penalty is the same for whatever product is used illegally, the users would always prefer to use the illegal version of product $A$ to illegal version of product $B^{37}$. The only case when developer $B$ would have the incentive to introduce the restriction of his services is, as we will see, when $X$ is "low," and A introduces strict restrictions of his services (low $\alpha$ ). In that case, the lack of developer $B$ implementing protection would result in the illegal usage of product $B$ (in this case, an illegal version of $B$ has quality $q_{B}$, while an illegal version of $A$ has $\alpha q_{A}$, which is lower than $\left.q_{B}\right)$. In other words, no user would use product $B$ legally unless developer $B$ also implements protection (see Case 2 below).

\subsubsection{Users set-up}

As in the previous chapter, we assume that every user has access to all the versions: to both legal versions $A, B$ and to the illegal versions of $A, B$ and decides based on the product prices and values. Utility for a user $\theta$ is then:

$$
U_{P}(\theta)=\left\{\begin{array}{lll}
\theta q_{i}-p_{i} & \ldots & \text { if he buys software. } \\
\theta \alpha q_{i}-X & \ldots & \text { if he uses software illegally. } \\
0 & \ldots & \text { if he does not use software at all. }
\end{array}\right.
$$

The important difference in Section 2 is developer $A$ cannot make any profit if he sets the price $p_{A}$ higher than $X$ because its legal product is eliminated from the market. In this section, on the other hand, there might be some users (top-end users with high $\theta$ ) that may prefer to buy the legal version rather than the restricted illegal one even if both versions

\footnotetext{
${ }^{37}$ In real life, the developer of a product with a lower quality competes strongly with an illegal version of a better product developer. Both developers know that introducing sophisticated protection could only discourage legal users from their services, while illegal users would always prefer to use a better product.
} 
(legal and illegal) are available and even when $X$ goes to zero. From utility function (11), we can identify 6 types of users indifferent between some two actions. Those users appear on the market under different levels of $X, q_{A}, q_{B}$, and $\alpha$. Only some of the indifferent users exist on a particular market but never all of them. Here are the 6 types of indifferent users:

1. $\begin{array}{lll}\theta_{P A} & \ldots & \text { The user indifferent between using legal product } A \text { and its illegal }\end{array}$ version.

2. $\theta_{0 P} \quad \ldots \quad$ The user indifferent between using illegal version $A$ and using nothing at all.

3. $\theta_{0 A} \quad \ldots \quad$ The user indifferent between using legal product $A$ and using nothing at all.

4. $\theta_{0 B} \quad \ldots$ The user indifferent between using legal product $B$ and using nothing at all.

5. $\theta_{B P}, \theta_{P B} \quad \ldots \quad$ The user indifferent between using legal product $B$ and using illegal version $A$.

6. $\theta_{B A} \quad \ldots \quad$ The user indifferent between using legal product $A$ and using legal product $B$.

In this chapter, we will again use the notation introduced in Notation 1 . As in the previous chapter, for a better illustration of the model behavior, we shall start with the monopoly case.

\subsection{Monopoly}

In the case of a monopoly market, developer $A$ can compete only with an illegal version of his own product. Similarly to the previous chapter, if the expected penalty $X$ is high enough that nobody is willing to use software illegally, we obtain the same market structure as in Section 1 (captured on Figure 1 ). This situation occurs when $\frac{1}{2} \bar{\theta} \alpha q_{A} \leq X$.

In the case where $X \leq \frac{1}{2} \bar{\theta} \alpha q_{A}$, there are users who prefer to use the illegal version and so setting $\alpha$ as low as possible is the right thing to do in order to increase the demand for the legal version In order to work out the monopolist's demand, we find user $\theta_{P A}$, who is indifferent between the legal and illegal product, and so this user is described by $\theta_{P A}=$ $\frac{p_{M}-X}{q_{A}-\alpha q_{A}}$. The demand for product $A$ is then $D_{A}=\left(\bar{\theta}-\theta_{P A}\right)$, and the monopolist profit is $\pi_{M}=\left(\bar{\theta}-\theta_{P A}\right) p_{M}$, while the demand for the illegal version is $D_{P}=\left(\theta_{P A}-\theta_{0 P}\right)$. Equilibrium price and profit are: 


$$
p_{M}^{*}=\frac{X+\bar{\theta} q_{A}(1-\alpha)}{2}, \pi_{M}^{*}=\frac{1}{4} \frac{\left(X+\bar{\theta} q_{A}(1-\alpha)\right)^{2}}{q_{A}(1-\alpha)} .
$$

This results in the distribution of users on the market as captured in Figure 6 .

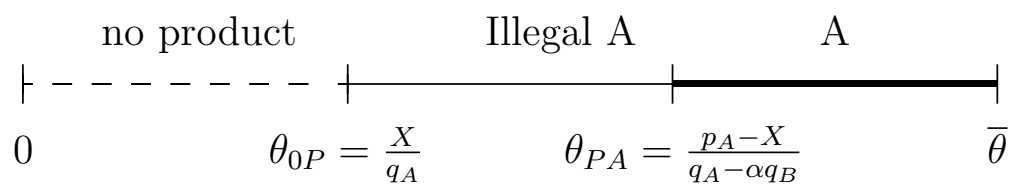

Figure 6: The decreased quality to illegal users on the monopoly market

Note that the monopolist that faces illegal usage but has an option to increase the number of legal users by restricting additional services generates uniformly higher profit than the monopolist that could only set $p_{M}^{*}=X$.

Clearly, now the profit of the monopolist increases not only in the level of expected penalty but also in the degree of restrictiveness to the additional services (that is, the lower the $\alpha$, the higher the monopolist's profit). Thus, the maximal restrictions of services to the illegal users are optimal requiring the minimum possible level of $\alpha$ that we label as $\underline{\alpha}$.

\subsection{Bertrand competition}

A user's decision to use an illegal version now again depends on the user's sensitivity to product quality $\theta$ as well as on the expected penalty $X$. We first focus on the situation in which only a developer of a higher quality product uses the restriction in services ${ }^{38}$. If $X$ is high enough such that $p_{B}<p_{A}<X$, then ${ }^{39}$ illegal usage is fully suppressed, and the market is divided between both developers, which is in fact, the case we have already analyzed in pure Bertrand competition in Section 2, see Figure 5. Assuming that illegal usage is not eliminated, and legal versions are on the market, then there are two interesting cases in which both developers operate on the market. The first one is $p_{B}<X<p_{A}$ and $q_{B}<\alpha q_{A}$ (implying $\frac{p_{B}}{q_{B}} \leq \frac{X}{\alpha q_{A}} \leq \frac{p_{A}}{q_{A}}$ ), and the second one is $X<p_{B}<p_{A}$ and $\alpha q_{A} \leq q_{B}$ (implying

\footnotetext{
${ }^{38}$ In the case that developer $B$ also introduces a restriction of, say, $\alpha_{B}$, then the product of developer $B$ will not be used illegally unless $\alpha_{B}$ is significantly higher than $\alpha$ resulting in $\alpha_{B} q_{B} \geq \alpha q_{A}$ as any user who decides on using an illegal product would use the illegal product of the highest available quality since the expected penalty is $X$ regardless of the product.

${ }^{39}$ In this part, whenever we write $p_{B}<p_{A}$ we mean $\frac{p_{B}}{q_{B}}<\frac{p_{A}}{q_{A}}$, which is a necessary condition for product $B$ to be in the market.
} 
$\left.\frac{X}{\alpha q_{A}} \leq \frac{p_{B}}{q_{B}} \leq \frac{p_{A}}{q_{A}}\right)$. In all other cases, either the legal version of product $B$ is eliminated so there is a monopoly for developer $A$, or the illegal usage of product $A$ is eliminated and there is pure Bertrand competition.

In the first case when $\frac{p_{B}}{q_{B}} \leq \frac{X}{\alpha q_{A}} \leq \frac{p_{A}}{q_{A}}$ and $q_{B} \leq \alpha q_{A}$, developer $A$ competes with an illegal version of his own product to capture users with relatively high $\theta$, while developer $B$ competes with an illegal version of product $A$ to capture users with relatively low $\theta$ (See Figure 7 ). In the second case, when $\frac{X}{\alpha q_{A}} \leq \frac{p_{B}}{q_{B}} \leq \frac{p_{A}}{q_{A}}$ and $\alpha q_{A}<q_{B}$, developer $A$ competes with developer $B$ for users with high $\theta$, while developer $B$ competes with the illegal version of $A$ for users with low $\theta$ (see Figure 8 ). The second case leads to a tougher competition between developers $A$ and $B$, where $q_{A}$ and $q_{B}$ are relatively close, while the first case describes a market where developer $B$ produces a significantly less valuable product than developer $A$, and thus, he can hardly compete with his legal version ${ }^{40}$.

\subsubsection{Case 1: Bertrand competition when $p_{B}<X<p_{A}$ and $q_{B}<\alpha q_{A}$, second stage}

$\left(\frac{p_{B}}{q_{B}} \leq \frac{X}{\alpha q_{A}} \leq \frac{p_{A}}{q_{A}}\right)$

This situation corresponds to a product distribution over the market in which there are three types of indifferent users:

1. A user indifferent between buying product $A$ and its illegal usage: $\theta_{P A}=\frac{p_{A}-X}{q_{A}-\alpha q_{A}}$,

2. A user indifferent between the illegal usage of product $A$ and buying product $B: \theta_{P B}=$ $\frac{X-p_{B}}{\alpha q_{A}-q_{B}}$, and

3. A user indifferent between buying product $B$ and not using any product at all: $\theta_{0 B}=$ $\frac{p_{B}}{q_{B}}$.

All users with $\theta \in\left(\theta_{B P}, \theta_{P A}\right)$ use an illegal version of product $A$. The users of the illegal version split the market into two sub-markets and to put it roughly, the illegal users recruit themselves from the middle part of the market. The profit function for each developer is then $\pi_{A}=\left(\bar{\theta}-\theta_{P A}\right) p_{A}=\left(\bar{\theta}-\frac{p_{A}-X}{q_{A}-\alpha q_{A}}\right) p_{A}$, and $\pi_{B}=\left(\theta_{B P}-\theta_{0 B}\right) p_{B}=$ $\left(\frac{X-p_{B}}{\alpha q_{A}-q_{B}}-\frac{p_{B}}{q_{B}}\right) p_{B}$. Equilibrium prices and profits are the following:

$$
p_{A}^{*}=\frac{\bar{\theta} q_{A}(1-\alpha)}{2}+\frac{X}{2}, p_{B}^{*}=\frac{q_{B}}{2 \alpha q_{A}} X
$$

\footnotetext{
${ }^{40}$ E.g., competition between the Microsoft Office 2010 package against small alternative developers such as 602 and its package known as "OpenOffice.org Software 602 Edition."
} 


$$
\pi_{A}^{*}=\frac{1}{4} \frac{\left(\bar{\theta} q_{A}(1-\alpha)+X\right)^{2}}{q_{A}(1-\alpha)}, \pi_{B}^{*}=\frac{1}{4} \frac{q_{B}}{\alpha q_{A}} \frac{1}{\alpha q_{A}-q_{B}} X^{2} .
$$

(F.O.C. and S.O.C. are stated in Appendix 1.3.1 ), and resulting market coverage is the following:

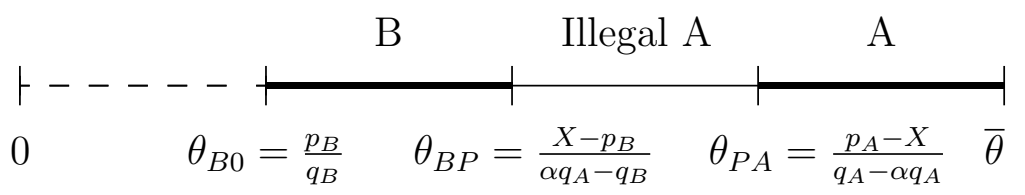

Figure 7: BC with illegal users in the middle of the market

Lemma 2 The only necessary and sufficient condition with respect to $X$ for this kind of equilibrium to exist is:

$$
0<X<X_{\alpha 1}=\frac{\bar{\theta} \alpha q_{A}\left(\alpha q_{A}-q_{B}\right)(1-\alpha)}{(2-\alpha) \alpha q_{A}-q_{B}}
$$

Proof. see Appendix 2.3.2

In this special case, only developer $A$ has the incentive to choose service restriction in the first stage. Moreover, note that the developers do not directly compete against each other because users who are using product $A$ illegally create a "buffer" between the legal users of products $A$ and $B$. Thus, the profit of each developer is independent on competitor's price and the driving factors of the profit are the level of the expected penalty $X$, and the level of restricted services $\alpha$. Moreover, note that the market coverage, equilibrium price, and, consequently, profit of developer $A$ are the same as if he was a monopolist constrained by $X \leq p_{A}^{*}$ (implying that $X \leq \frac{1}{2} \bar{\theta} \alpha q_{A}$, see sub-section 3.2 ).

Remark 4 Developer $A$ 's decision to implement $\alpha$ and then set the price to $p_{A}=X$ is never optimal in the given set-up.

Lemma 3 In the case of duopoly competition when $\frac{p_{B}}{q_{B}} \leq \frac{X}{\alpha q_{A}} \leq \frac{p_{A}}{q_{A}}$ and $q_{B} \leq \alpha q_{A}$, the equilibrium profit and price of developer $A$ as well as developer $B$ are decreasing in $\alpha$ as long as $q_{B} \leq \alpha q_{A}$ holds.

Proof. The behavior of $p_{A}^{*}(\alpha), p_{B}^{*}(\alpha), \pi_{B}^{*}(\alpha)$ can be seen immediately from equilibrium (13) and (14), proof that $d \pi_{A}^{*}(\alpha) / d \alpha<0$ could be also easily derived. 
Intuitively as $\alpha$ decreases, the illegal usage becomes more costly and consequently shrinks. Since both developers compete directly only with an illegal version of product $A$, this improves their competitive advantage by making legally accessible quality more attractive compared to the illegal one allowing in turn both prices to increase in equilibrium.

\subsubsection{Case 2: Bertrand competition when $X<p_{B}<p_{A}$ and $q_{B}>\alpha q_{A}$, second stage \\ $\left(\frac{X}{\alpha q_{A}} \leq \frac{p_{B}}{q_{B}} \leq \frac{p_{A}}{q_{A}}\right)$}

Note that in this set-up, developer $B$ would also be forced to introduce the IPR protection of $\alpha$ in order to stay in the market. Otherwise the users who do not buy a legal version of product $A$, would prefer to use the illegal version of product $B$, whose quality would be $q_{B}>\alpha q_{A}$. As a consequence of IPR implementation by both developers, there would be a direct competition between the two developers, but their payoffs depend on the level of $X$ and the developers' IPR protection $\alpha$. A user indifferent between $A$ and $B$ is $\theta_{B A}=\frac{p_{A}-p_{B}}{q_{A}-q_{B}}$, and a user indifferent between illegal usage of $A$ and buying $B$ is $\theta_{P B}=\frac{p_{B}-X}{q_{B}-\alpha q_{A}}$. Users with $\theta \in\left(\frac{X}{\alpha q_{A}}, \frac{p_{B}-X}{q_{B}-\alpha q_{A}}\right)$ use an illegal version of product $A$. The profits for developers are: $\pi_{A}=\left(\bar{\theta}-\theta_{B A}\right) p_{A}$ and $\pi_{B}=\left(\theta_{B A}-\theta_{P B}\right) p_{B}$. This situation leads to the following distribution on the market:

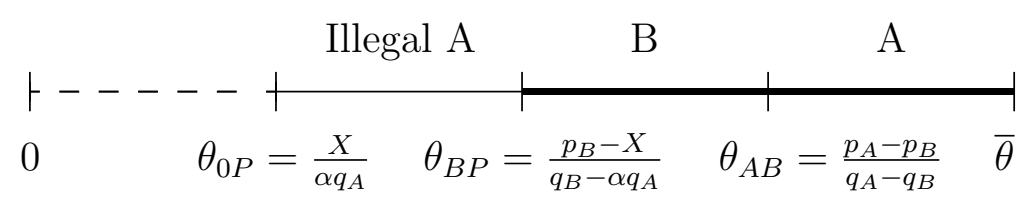

Figure 8: BC with illegal users at the low end of the market

This results in the following equilibrium prices and profits:

$$
\begin{gathered}
p_{A}^{*}=\left(q_{A}-q_{B}\right) \frac{\bar{\theta} 2 q_{A}(1-\alpha)+X}{4 q_{A}-3 \alpha q_{A}-q_{B}}, p_{B}^{*}=\left(q_{A}-q_{B}\right) \frac{\bar{\theta}\left(q_{B}-\alpha q_{A}\right)+2 X}{4 q_{A}-3 \alpha q_{A}-q_{B}} . \\
\pi_{A}^{*}=\left(q_{A}-q_{B}\right)\left(\frac{2 \bar{\theta} q_{A}(1-\alpha)+X}{4 q_{A}-3 \alpha q_{A}-q_{B}}\right)^{2}, \\
\pi_{B}^{*}=\left(q_{A}-q_{B}\right)(1-\alpha) q_{A} \frac{\left(\bar{\theta}\left(q_{B}-\alpha q_{A}\right)+X\right)^{2}}{\left(q_{B}-\alpha q_{A}\right)\left(4 q_{A}-3 \alpha q_{A}-q_{B}\right)^{2}} .
\end{gathered}
$$


Lemma 4 A necessary condition for the existence of an equilibrium is satisfied only for $X$ and $\alpha$ such that:

$$
0 \leq X_{\alpha 2}=\frac{\bar{\theta}\left(q_{A}-q_{B}\right)\left(q_{B}-\alpha q_{A}\right) \alpha q_{A}}{4 q_{A} q_{B}-q_{B}^{2}-2 \alpha q_{A}^{2}-\alpha q_{A} q_{B}} .
$$

Proof. see Appendix 2.3.3

Lemma 5 The equilibrium profit and price of developer $A$ as well as developer $B$ are decreasing in $\alpha$ when $q_{B}>\alpha q_{A}$ holds.

Recall that the competition in Case 2 is tougher than in Case 1 since developers now compete directly with each other, and the increase in market share of one developer automatically implies a decline in the share of the other developer.

\subsubsection{Stackelberg competition}

In Case 1 above, the illegal version of product $A$ serves as a "buffer" between the two legal products, and the prices chosen by the developers do not depend on each other, i.e., the reaction functions $p_{i}\left(p_{j}\right)$ do not depend on $p_{j}$. Therefore, the Stackelberg outcome in this case is the same as the Bertrand outcome above. As for Case 2, the Stackelberg outcome is in Appendix 2.4 .

\subsection{Optimal service restrictions: the first stage}

The optimal service restriction is rather simple in our set-up given the assumption of no costs for restraining services. (Recall that profit functions in both Cases 1 and 2 decrease in $\alpha$.) Thus, the optimal service restriction is always such that $\alpha^{*}=\underline{\alpha}$ irrespective of the level of $X$ (provided that the size of $X$ is such that it requires the imposition of a service restriction by at least one developer, that is, $X<p_{A}$ ). What is more interesting here is to see how the levels of optimal $\alpha$ and $X$ affect the emerging market structure and market coverage in the second stage equilibrium. We start with the buffer case: Case 1.

\subsection{1 $\quad \alpha^{*}=\underline{\alpha}$, and $p_{B}<X<X_{\alpha 1}$}

This case appears in equilibrium when $\alpha$ is relatively large $\left(\alpha^{*} q_{A}>q_{B}\right)$, and this is typically the case when the quality of the first developer is "substantially" larger than the quality of 
the second developer. The interesting (comparative static) question to ask here is what would happen if the regulator increases $X$ to be at $X_{\alpha 1}$ or larger. If $X$ exceeds $X_{\alpha 1}$, then piracy becomes too costly and, consequently, the buffer of illegal users is completely eliminated (that is, $\underline{\alpha} q_{A} \bar{\theta}-X<0$ for all $\theta$ ). Thus an expected penalty high enough (such that $X>X_{\alpha 1}$ ), restores pure Bertrand competition, and so, a pair of private and government protections $\{\underline{\alpha}$, $\left.X_{\alpha 1}\right\}$ are able to restore the competition without illegal users. Recall that without private protection, the regulator would have to set a much higher excepted penalty to achieve the same outcome (that is, $X$ has to be such that $X>p_{A}$ ).

An alternative way in which the pure Bertrand competition would appear in equilibrium is the situation when $\underline{\alpha} q_{A}=q_{B}$ (or $\underline{\alpha} q_{A}$ is "close" enough to $q_{B}$ ). The intuition is similar to the one above; the usage of an illegal version becomes non-attractive when $\alpha$ falls so low that a legal version of product two has the same (or only slightly lower) value for consumers but is offered at a lower price, $p_{B}<X$. Thus, again the illegal usage is completely eliminated.

\subsection{2 $\quad \alpha^{*}=\underline{\alpha}$, and $X<p_{B}<X_{\alpha 2}$}

Clearly, this situation appears when $\underline{\alpha}$ is relatively small so that $\underline{\alpha} q_{A}<q_{B}$, and there is direct duopoly competition (Case 2 above) in which illegal usage occurs only at the lowest tail of the market. This would likely be the case when quality of the first developer is not "much" larger than the quality of the second developer. Note that it would be now optimal for both developers to introduce service restrictions. Moreover, both developers choose the technically minimal possible $\alpha$, i.e., $\alpha_{A}^{*}=\alpha_{B}^{*}=\underline{\alpha}$. Let us assume again that the regulator sets the expected penalty that exceeds the critical value for Case 2 to occur, (that is, $X>X_{\alpha 2}$ ). In that case, $\frac{X}{\alpha q_{A}}>\frac{p_{B}}{q_{B}}$ (or $\theta_{0 P}>\theta_{0 B}$ ) implying that no one would use an illegal version and again, the competition would be back to the pure Bertrand. Thus, in this set-up too, a pair of $\left(\underline{\alpha}, X_{\alpha 2}\right)$ restores a pure Bertrand competition and much like in the case of 3.4.1 above, $X_{c 2}$ is substantially lower than the expected penalty that would alone achieve complete elimination of illegal usage.

So in both cases, private and government IPR protection are substitutes in the sense that introducing private protection in the form of service restrictions, enables the regulator to eliminate the illegal usage of software with a much lower (and less costly) expected penalty.

Finally, if we alternatively assume that:

1. It would be costly for the developers to incur service restrictions, 
2. Optimal $\alpha$ can be anywhere in the interval $(0,1)$, and

3. The corresponding cost function for implementing $\alpha, C(\alpha)$ is convex enough to generate an interior maximum, then $\alpha^{*}$ can be such that either $\alpha^{*} q_{A}>q_{B}$ or $\alpha^{*} q_{A}<q_{B}$ depending on the size of $X$ and the shape of the cost function, $C(\alpha)$ such that $\frac{\partial}{\partial \alpha} C(\alpha)>0$ and $\frac{\partial^{2}}{\partial \alpha \partial \alpha} C(\alpha)>0$.

\subsection{Key section results}

In the case of the monopoly set-up, we show that developer $A$ competes against an illegal version of his own product, and it is optimal for him to maximally restrict the additional software services for illegal users. He always operates in the market and is always better off than the monopolist that sets the price to an expected penalty when faced with illegal usage (as in Section 1).

In the case of Bertrand competition, the interaction between the developers' and the regulator's IPR (as well as the nature of the competition) depends critically on whether IPR protection, $X$, is "High," "Medium," or "Low."

1. A "High" expected penalty can be defined as the one in which $p_{B} \leq p_{A} \leq X$. In this case, the developers' IPR protection is redundant, and obviously the regulator's IPR protection acts as a substitute for the developers' IPR.

2. The regulator IPR protection is "Medium" if $p_{B} \leq X \leq p_{A}$. In that case, we see that the situation of the highest interest is the one where $\frac{p_{B}}{q_{B}} \leq \frac{X}{\alpha q_{A}} \leq \frac{p_{A}}{q_{A}}$ and $q_{B}<\alpha q_{A}$. We call it a buffer case. Developer $A$ earns the same profit as a (constrained) monopoly that is positively affected by the level of the expected penalty $X$. The optimal service restriction is always such that $\alpha^{*}=\underline{\alpha}$ irrespective of the size of $X$.

3. Finally, there is a third case of "Low" IPR protection, in which $X \leq p_{B} \leq p_{A}$. The most interesting situation occurs when $\frac{X}{\alpha q_{A}} \leq \frac{p_{B}}{q_{B}} \leq \frac{p_{A}}{q_{A}}$ and $\alpha q_{A}<q_{B}$. In this situation developers, compete directly against each other, and the level of $X$ positively affects the profit functions of both developers. Moreover, developer $B$ has also to introduce the restriction of services to the degree $\alpha_{B}$ for the illegal users who would otherwise prefer to use product $B$ illegally. The developers choose the maximal possible level of IPR protection $(\alpha=\underline{\alpha})$. 
4. Marginal changes in $X$ do not affect optimal choices of $\alpha$ given that the costs of the service restriction are zero. If, on the other hand, these costs are substantial yielding the inverse U-shaped profit function and interior maximum for $\alpha$, then the marginal change in $X$ does affect the optimal choice of service restriction.

5. Finally in both cases, private and government IPR protection are substitutes since the introduction of private protection enables the regulator to eliminate the illegal usage of software with a lower expected penalty.

6. In the case of the Stackelberg competition, we show that developer $B$ has no advantage from setting his price as the second one in the "buffer" case. There is a "second mover advantage," only in the case of direct competition like in Case 2 above.

\section{Physical product protection against copying}

In this section, we focus on a situation where the developers can eliminate illegal usage by implementing physical protection against copying. By physical protection we understand that installing an illegal version of the software is more difficult either because of a low availability of the illegal version or because of the high requirement on the users' skill to install (or use) the illegal version. An example of such a protection is the DVD with games where a version coming from standard copying with a DVD burner cannot be installed on a PC any longer ${ }^{41}$. Another example is requiring users to authenticate their copy on the developer's web pages during installation, which could be technically complicated to avoid (e.g., only by installing a "crack" to a particular directory and a set of steps to complete the installation). All such tools create obstacles in installing an illegal version, and thus limit its availability to common users. After installation, however, a user often may not distinguish an illegal version from the legal one. As already mentioned in the introduction, some forms of DRM can also serve as examples of such a protection. Thus, a user's perception of software quality is often intact.

As noted in the introduction, most of the research papers on IPR protection in software markets have analyzed the trade-off between the perfect protection and the costs of its

\footnotetext{
${ }^{41}$ The illegal copy is not working since the original DVD is intentionally produced with certain kinds of mistakes, and during copying, these mistakes are always corrected by the "burning" software. At the same moment, during the installation process, those mistakes are mandatory for the successful completion of the installation.
} 
implementation. In this section, we focus on what impact the size of the expected penalty $X$ has on the developers incentives to introduce some kind of physical protection against illegal copying. We also study the impact of such protection on mutual competition between developers.

\subsection{Model set-up}

\subsubsection{Developers' problem}

We assume now that both developers have access to technology that allows product protection against copying and illegal usage ${ }^{42}$. The developers' decisions are dependent only on the profitability of such a step. The protection against copying is imperfect, which means that a fraction of the users still have access to the illegal version ${ }^{43}$. This fraction of users is uniformly distributed over the whole interval $\langle 0, \bar{\theta}\rangle$. We say that a developer implements protection at level $c$, if for each $\theta \in\langle 0, \bar{\theta}\rangle$ the fraction of users with the ability to use the illegal version is $(1-c)$, and the remaining fraction of users $(c)$ could only use the legal version. Protection $c$ is from interval $\langle 0,1\rangle$, and if $c$ tends to 1 we say that protection becomes perfect, while $c$ tending to 0 represents the full public availability of an illegal version ${ }^{44}$. We further assume that both developers could implement this kind of protection, and that they could differ among themselves in the protection level $c$. Much like in the previous section, we can think about a two-stage game in which one or both developers choose the level of private protection in the first stage, and then they compete in prices in the second stage.

Unlike in the case of restricting services to illegal users, it is now reasonable to assume that implementing physical protection is costly, and that these costs rise more than proportionally as $c$ increases tending to infinity as $c$ approaches 1 . Thus, the costs of implementing protection $c$, labelled as $C=h(c)$, possess the following properties:

1. $h(0)=0, \lim _{c \rightarrow 1} h(c)=+\infty$;

2. $\frac{\partial}{\partial c} h(0)=0, \frac{\partial}{\partial c} h(c)>0$

\footnotetext{
${ }^{42}$ Neither legal nor licence restrictions are assumed for the developer in the case of implementing protection against copying.

${ }^{43}$ By eliminating public availability we mean both no access to an illegal version or access to an illegal version accompanied by the limited user's skill to install/use the illegal version.

${ }^{44}$ The availability of an illegal version and the ability to break it differs significantly among users and is more dependent on technical skill than on the sensitivity to price $\theta$. The uniform distribution is an analytical simplification not harming the nature of the essay.
} 
3. $\frac{\partial^{2} h(c)}{\partial c^{2}}>0$ and

4. $\Pi_{i}^{*}=\pi_{i}^{*}\left(c_{i}\right)-h\left(c_{i}\right)$ is a concave function reaching its maximum at $c_{i}^{*} \in(0,1)$. (We use the symbol $\Pi$ for net profit, when protection costs are accounted for, while $\pi$ stands for the price-competition stage profit.)

Note that much like in the previous section with restricting services, we are not so interested in the actual optimal value of protection, $c$ but rather in its interaction with the expected penalty $X$ and, consequently, its impact on equilibrium prices, profits, and market coverage.

\subsubsection{The consumer problem}

Recall that in the previous sections, all users have access to illegal versions, and the user's decision to use an illegal version was always based on the expected utility coming from usage of such a version compared to the utility from using a legal version. In this section, we assume that only some users have access to both a legal and an illegal version, while some users have access only to a legal version. The users with access to both versions prefer the legal version only if the utility from it is higher and their proportion is $1-c$. The utility function of user $\theta$ is the following:

$$
U_{P}(\theta)=\left\{\begin{array}{lll}
\theta q_{i}-p_{i} & \ldots & \text { if he buys the legal version of the software. } \\
\theta q_{i}-X & \ldots & \text { if he uses the software illegally. } \\
0 & \ldots & \text { if he does not use the software at all. }
\end{array}\right.
$$

Users without access to the illegal version could compare only the expected utility from purchasing the legal version and not using it at all. Their proportion is $c$, and the utility function of user $\theta$ is:

$$
U_{P}(\theta)=\left\{\begin{array}{lll}
\theta q_{i}-p_{i} & \ldots & \text { if he buys the legal version of the software } \\
0 & \ldots & \text { if he does not use the software at all. }
\end{array}\right.
$$

\subsubsection{The market environment}

As we already noted, both developers could implement physical protection for their product, and so three basic combinations of product protection could occur in the market : 
1. None of the developers implement protection. This situation arises when $X$ does not bind in the maximization problems of either $A$ or $B$ so that in the equilibrium, we have $p_{B}^{*} \leq p_{A}^{*} \leq X$.

2. Developer $A$ implements protection while developer $B$ does not. This situation occurs when pure Bertrand equilibrium is not possible because $X$ would be binding for developer $A$ since $p_{B}^{*} \leq X \leq p_{A}^{*}$.

3. Both developers implement protections. ${ }^{45}$ Finally for low $X$, both developers would have to introduce protection since pure Bertrand equilibrium would result in $X \leq$ $p_{B}^{*} \leq p_{A}^{*}$.

Before analyzing the above cases in more detail, we, as in the previous two sections, first start with the monopoly case that helps us to illustrate the flavor of the model.

\subsection{Monopoly}

As in the previous two sections, we start with a monopoly case that will help us to illustrate the flavor of the model. Consider now developer $A$ who introduces a level of protection at $c$ for his product $q_{A}$ and sets the price $p_{M}$. In analyzing monopolist behavior, we could focus only on the case when the expected penalty is such that $X<p_{M}$, since the case where $X>p_{M}$ is already described in the Section 2, and in this case, no user has the incentive to use an illegal version. Users' demand for the legal product of monopoly developer $A$ is $D_{A}=c\left(\bar{\theta}-\frac{p_{M}}{q_{A}}\right)$ and leads to the following market coverage:

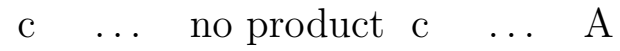

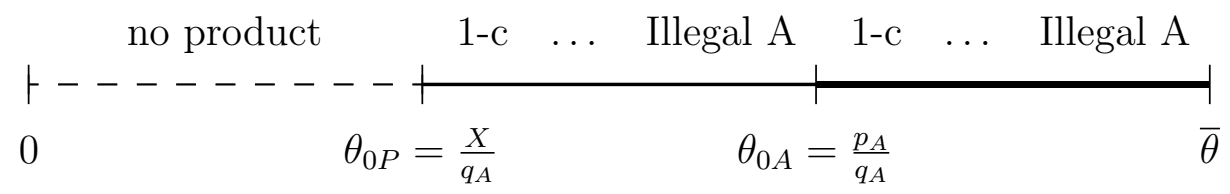

Figure 9: Monopoly market with product protection $c$

\footnotetext{
${ }^{45}$ Note that the case in which only developer $B$ implements protection never occurs. If $B$ has to implement protection due to the low expected penalty $X$, then developer $A$ must also implement physical protection because his product would be the primary target of illegal usage.
} 
Monopoly equilibrium is analogous to the one in Section 1, as could be immediately derived from $(2)$ :

$$
p_{M}^{*}=\frac{1}{2} \bar{\theta} q_{A}, \pi_{M}^{*}=c \frac{1}{4} \bar{\theta}^{2} q_{A} .
$$

Note that under the assumptions regarding $h(c), \Pi_{M}^{*}=\pi_{M}^{*}-h(c)$ has a unique maximum, $c_{M}^{*} \in(0,1)$. A monopoly developer $A$ always has an option to decrease the price to $X$ instead of implementing protection $c$. By comparing developer $A$ 's profit in the case of lowering the price to $X$ as in Section 2, see monopolist profit in (3), with his profit after implementing protection, we find out that developer $A$ prefers physical protection as long as the expected penalty, $X$, is below a certain critical level. More specifically, even with protection costs $h(c)=0$, it is more profitable to lower the price to $X$ instead of implementing protection if $X>\bar{\theta} q_{A} \frac{1-\sqrt{1-c_{M}^{*}}}{2}$.

\subsection{Bertrand competition}

As in the previous part, we omit the case when the expected penalty $X$ is high enough $\left(p_{B}^{o} \leq\right.$ $\left.p_{A}^{o} \leq X\right)$, and developers have no incentives to introduce physical protection against copying (this case we already analyzed in Section 2). In analyzing this set-up, first, we focus on the case where only developer $A$ has the incentive to introduce protection $p_{B}^{*} \leq X \leq p_{A}^{*}$ and then, finally, on the case where both developers have such incentives, that is, $X \leq p_{B}^{*} \leq p_{A}^{*}$. Note that in our set-up, prices are as usually strategic complements (see Tirole, 1989, and Bulow et al., 1985), that is, $\frac{\partial^{2} \pi_{i}}{\partial p_{B} \partial p_{A}}>0$.

\subsubsection{Only developer $A$ implements protection $c$}

In this case, where $p_{B}^{*} \leq X \leq p_{A}^{*}$, only developer $A$ has the incentive to implement physical protection since the product of developer $B$ would be used only legally. As we already mentioned in our model set-up, the illegal version of product $A$ is available only to the fraction $1-c$ of the users' base. Product $A$ is used illegally only by users with $\frac{X}{q_{A}} \leq \theta$, while users with $\theta \leq \frac{X}{q_{A}}$ prefer not to use the product at all. The demand for product $B$ consists of users with low sensitivity $\theta$ to purchasing product $A$, who, at the same time, have no access to an illegal version of $A$, but their $\theta$ is high enough to buy product $B$. These users have $\theta \in\left(\frac{p_{B}}{q_{B}}, \frac{p_{A}-p_{B}}{q_{A}-q_{B}}\right)$, and their fraction is $c$. As for the users with access to an illegal version of product $A$, there are two sub-cases that could occur in equilibrium depending on the size of the expected penalty: 
1. The first sub-case occurs when there are some users who have illegal access to product $A$ but still want to buy product $B$, or more formally, the measure of these users is strictly positive with $\theta \in\left(\frac{p_{B}}{q_{B}}, \frac{X-p_{B}}{q_{A}-q_{B}}\right)$, and so, $\frac{X-p_{B}}{q_{A}-q_{B}}>\frac{p_{B}}{q_{B}}$. These users would like to purchase product $B$ if $X$ is not so "low" (in the sense that $X>p_{B} \frac{q_{A}}{q_{B}}$ ). The market coverage is given in Figure 10 .

2. The second sub-case occurs when illegal users always prefer an illegal version of $A$ to the legal version of $B$, that is, when $\theta q_{A}-X>\theta q_{B}-p_{B}$ for all $\theta$ since illegal usage is then more profitable even for the consumer with the lowest valuation. So, $X$ has to be "low" enough, that is, $\frac{X-p_{B}}{q_{A}-q_{B}} \leq \frac{p_{B}}{q_{B}}$ (or equivalently $X \leq p_{B} \frac{q_{A}}{q_{B}}$ ) given that $p_{B}^{*} \leq X$ still holds. The market coverage of this case is presented in Figure 11 .

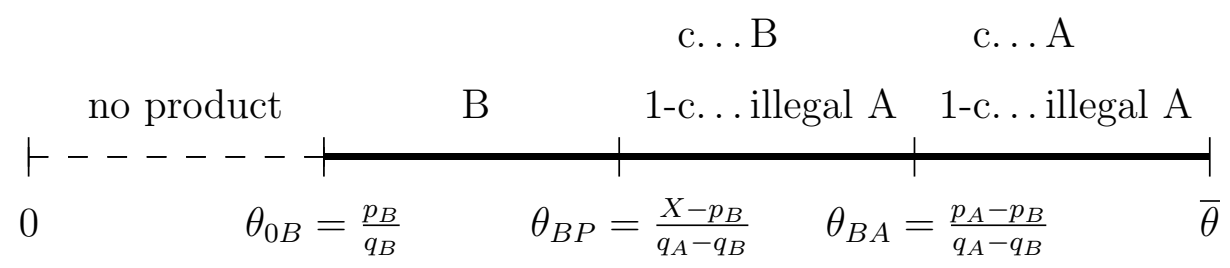

Figure 10: BC, when developer $A$ introduces protection $c$ (Case 1).

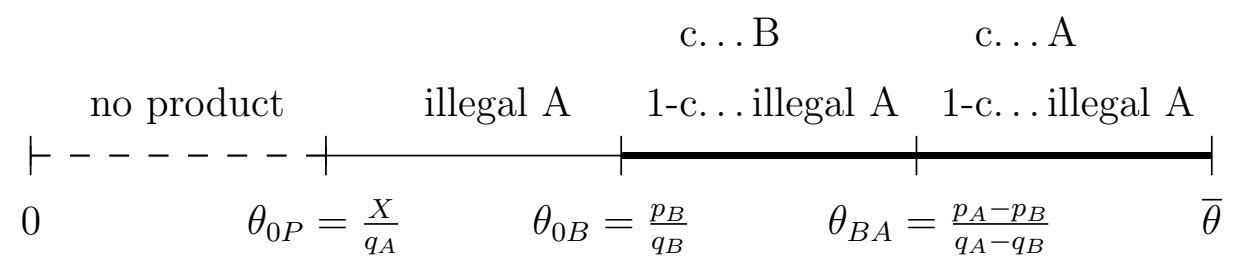

Figure 11: BC, when developer $A$ introduces protection $c$ (Case 2).

As for Sub-case 1, we obtain demand for legal versions of both products by putting all fraction of users together:

$$
\begin{aligned}
D_{A} & =c\left(\bar{\theta}-\frac{p_{A}-p_{B}}{q_{A}-q_{B}}\right), \\
D_{B} & =c\left(\frac{p_{A}-p_{B}}{q_{A}-q_{B}}-\frac{p_{B}}{q_{B}}\right)+(1-c)\left(\frac{X-p_{B}}{q_{A}-q_{B}}-\frac{p_{B}}{q_{B}}\right)= \\
& =\frac{c p_{A}+(1-c) X-p_{B}}{q_{A}-q_{B}}-\frac{p_{B}}{q_{B}} .
\end{aligned}
$$


In Sub-case 2, only the users without access to an illegal version of $A$ buy product $B$ so the demand functions are now:

$$
\begin{aligned}
& D_{A}=c\left(\bar{\theta}-\frac{p_{A}-p_{B}}{q_{A}-q_{B}}\right), \\
& D_{B}=c\left(\frac{p_{A}-p_{B}}{q_{A}-q_{B}}-\frac{p_{B}}{q_{B}}\right) .
\end{aligned}
$$

Note that Sub-case 2 is practically identical to the pure Bertrand case yielding the same equilibrium prices, see (4), and yielding the same market coverage as well as the equilibrium profits that are only sized down by factor $c$, see (5). Most importantly, small changes in the expected penalty have no impact on the size of optimal private protection nor on the other equilibrium values.

Thus, we focus on the more interesting Subcase 1. We start with determining the range of the expected penalty values $X$ such that this sub-case is the Nash equilibrium in prices. Namely, sub-case 1 is not an equilibrium if (i) at least one developer's profit, given the other developer's price choice, does not have a local maximum in the relevant price range. Moreover, it is also not an equilibrium if (ii) there is a local maximum in the relevant range, but at least one developer is better off deviating to a price outside the range (e.g., developer $A$ can be better off deviating to $p_{A}=X$ ). Intuitively for developer $A$ to charge a high price $p_{A}>X$, the value of $X$ should be small enough so that developer $A$ prefers introducing protection than to simply lowering the price to $X$. As for developer $B$ to charge a low price $p_{B}<X \frac{q_{B}}{q_{A}}, X$ should be large enough so that developer $B$ prefers charging a low price to both charging an intermediate price $X \frac{q_{B}}{q_{A}} \leq p_{B} \leq X$ and introducing protection or charging a high price $p_{B}>X$.

For (i), we show in Appendix 3.3.4 that a necessary condition on $X$ is $X_{c l}<X<X_{c u}$, where $X_{c l}=\frac{\bar{\theta} c q_{A}\left(q_{A}-q_{B}\right)}{2(1+c) q_{A}-c q_{B}}$, and $X_{c u}=2 \bar{\theta} q_{A} \frac{q_{A}-q_{B}}{4 q_{A}-q_{B}}$; note that the upper bound $X_{c u}$, intuitively, coincides with the equilibrium price $p_{A}^{o}$ from the case of the pure Bertrand equilibrium (4). Then both developers' profits reach the internal local maxima in the price ranges corresponding to our sub-case, with the prices equal to

$$
p_{A}^{*}=\frac{X(1-c) q_{B}+2 \bar{\theta} q_{A}\left(q_{A}-q_{B}\right)}{4 q_{A}-c q_{B}}, p_{B}^{*}=q_{B} \frac{2 X(1-c)+\bar{\theta} c\left(q_{A}-q_{B}\right)}{4 q_{A}-c q_{B}} .
$$

For (ii), we verify that neither developer has an incentive to unilaterally deviate given that the other developer sets the equilibrium price, $p_{i}^{*}$. For developer $A$, it can be profitable to deviate to $p_{A}=X$ (given that developer $B$ sets $p_{B}^{*}$ ) if the decrease in price from $p_{A}^{*}$ 
to $X$ is more than compensated for by an increase in the number of consumers that is no longer confined to fraction $c$, and for $X$ large enough, such a deviation would yield a higher profit even without protection costs, $h(c)=0$. As for developer $B$, if $p_{B}^{*}$ is close enough to $X \frac{q_{B}}{q_{A}}$, then it may pay off to jump to a higher price $p_{B} \in\left(X \frac{q_{B}}{q_{A}}, X\right)$ given that developer $A$ sets $p_{A}^{*}$ as in this case, the effect of such a price increase would more than offset the loss of the consumer base. The analysis in Appendix 3.3.4 shows that for an equilibrium to exist, $X$ should not be "too large" for developer $A$, so that $X<X_{c}^{+}<X_{c u}$, nor should it be "too small" for developer $B$, so that $X>X_{c}^{-}>X_{c l}$. While values $X_{c l}$ and $X_{c u}$ always define a non-empty range, the condition $X_{c}^{-}<X<X_{c}^{+}$defines a non-empty set only if $c>\frac{\sqrt{5}-1}{2} \approx 0.618034$, and if the quality ratio is not too high ${ }^{46}$, then the lower bound on $c$ can be improved to $c>\underline{c} \approx 0.704402$. If $X \in\left(X_{c}^{-}, X_{c}^{+}\right)$, then none of the developers have an incentive to deviate, and the prices above constitute an equilibrium.

As for the comparative statics analysis with respect to $c$, it is straightforward to show that equilibrium prices $p_{A}^{*}(c), p_{B}^{*}(c)$ and the profit $\pi_{B}^{*}(c)$ increase as the level of physical protection $c$ increases, so developer $A$ acts strategically and softens the price competition and (in jargon) displays pacifistic "fat cat" behavior (see Fudenberg and Tirole, 1984).

We now focus on our key issue of how private and public protection interact. More specifically, we study the effect of the expected penalty $X$ on the optimal developer's protection, $c^{*}$. The direction of this effect is determined by the impact of the expected penalty on the marginal profitability of private protection (more technically, on the sign of $\frac{\partial^{2} \pi_{A}^{*}}{\partial c \partial X}$ ) and also by the existence of the interval $\left(X_{c}^{-}, X_{c}^{+}\right)$. As we stated above, the necessary condition for that interval to be non-empty is that $c^{*}>\frac{\sqrt{5}-1}{2}$, and this, in turn, implies (or is sufficient for) $\frac{\partial^{2} \pi_{A}^{*}}{\partial c \partial X}<0$ entailing that the rise in the expected penalty decreases marginal profitability. This situation is described in jargon as "strategic substitutability" between $c^{*}$ and $X$ so that $\frac{d c^{*}}{d X}<0$.

Proposition 1 Private and public protection are always strategic substitutes, that is, $\frac{d c^{*}}{d X}<$ 0 .

Proof. see Appendix 3.3.5

\footnotetext{
${ }^{46}$ Here "not too high" means that $q_{B} / q_{A}$ is below the threshold value, which is itself above 0.9 , so we can be almost sure that this is the case and consider it as the general situation.
} 
With $c^{*}$ being "large," the "cost effect" dominates the effect on revenue in the sense that the gains of additional public protection are lower than the ensuing private costs of protection. So developer $A$ cuts back his private protection in response to the increased public protection, decreasing thus its private protection costs, and harming developer $B$ (recall that $\frac{d \pi_{B}^{*}(c)}{d c}>0$ ).

The nature of the interaction between the private and public IPR protection enables us to further study the comparative statics effects of $X$ on equilibrium prices and profits.

Lemma 6 The effect of $X$ on $p_{A}^{*}(X)$ and $p_{B}^{*}(X)$ is a priori undetermined.

Proof. Note that $\frac{d p_{i}}{d X}(c(X), X)=\frac{\partial p_{i}}{\partial c} \frac{d c}{d X}+\frac{\partial p_{i}}{\partial X}$. Straightforward differentiation shows that direct effect of $X$ on prices is positive, that is, $\frac{\partial p_{i}}{\partial X}>0$. From the analysis above, we know that $\frac{\partial p_{i}}{\partial c}>0$, but $\frac{d c}{d X}<0$. Thus, the indirect effect, $\frac{\partial p_{i}}{\partial c} \frac{d c}{d X}<0$.

Lemma 7 The effect of $X$ is positive on $\pi_{A}^{*}(X)$ but the respective effect on $\pi_{B}^{*}(X)$ is a priori unclear.

Proof. Note that $\frac{d \pi_{A}^{*}(X)}{d X}(c(X), X)=\frac{\partial \pi_{A}^{*}}{\partial X}>0$. Note further that $\frac{d \pi_{B}^{*}(X)}{d X}(c(X), X)=$ $\frac{\partial \pi_{B}^{*}(X)}{\partial c} \frac{d c^{*}}{d X}+\frac{\partial \pi_{B}^{*}}{\partial X}$, where $\frac{\partial \pi_{B}^{*}(X)}{\partial c} \frac{d c}{d X}<0$ since $\frac{d c^{*}}{d X}<0$ and $\frac{\partial \pi_{B}^{*}}{\partial X}>0$. Thus, the direct and indirect effects have a conflicting impact on developer B's profit.

As we see, developer $A$ reacts aggressively on an increase in $X$ and cuts back in his private protection in response to increased public protection. As for developer $B$, if the net outcome of the above two conflicting (direct and indirect) effects is negative, the profit of developer $B$ and equilibrium prices fall making price competition tougher. As a result, a "fat cat" strategy in this case becomes a bit diluted due to the enhanced public protection while, on the other hand, consumers of both goods benefit due to the decrease in equilibrium prices $^{47}$.

\subsubsection{Both developers $A$ and $B$ implement protection}

If the regulator sets up a very low expected penalty $\left(X \leq p_{B}^{*} \leq p_{A}^{*}\right)$, then, naturally, both developers have to either implement physical protection or decrease prices to $X$; otherwise, they would be out of the market.

\footnotetext{
${ }^{47}$ It is straightforward to show that entry deterrence by means of $c$ is not feasible in the set-up under consideration.
} 
We denote protection used by developer $A$ as $c_{A}$ and protection used by developer $B$ is $c_{B}$. Further, we assume that users may have access either to an illegal version of product $A$, an illegal version of product $B$, or to both illegal versions. Moreover, we assume that access to an illegal version of product $A$ and $B$ are mutually independent so there are users on the market that have access to illegal versions of product $A$ but not to illegal versions of product $B$ and vice versa. Then there are the following fractions of users on the market:

1. $c_{A} c_{B}$

2. $c_{A}\left(1-c_{B}\right)$

3. $\left(1-c_{A}\right) c_{B}$

4. $\left(1-c_{A}\right)\left(1-c_{B}\right) \quad \ldots \quad$ The fraction of users with access to illegal versions of both products.

We have now the following types of users:

1. $\theta \in\left(\frac{p_{A}-p_{B}}{q_{A}-q_{B}}, \bar{\theta}\right) \quad \ldots$ Users who buy product $A$ if they do not have access to any illegal version;

2. $\theta \in\left(\frac{p_{B}}{q_{B}}, \frac{p_{A}-p_{B}}{q_{A}-q_{B}}\right) \quad \ldots \quad$ Users who buy product $B$ if they do not have access to an illegal version of $A$;

3. $\theta \in\left(\frac{X}{q_{A}}, \bar{\theta}\right) \quad \ldots$ Users who use an illegal version of $A$ if they have access to it;

4. $\theta \in\left(\frac{p_{A}-X}{q_{A}-q_{B}}, \bar{\theta}\right) \quad \ldots$ Users who buy $A$ if they have access only to an illegal version of $B$.

Given the above set-up, it seems that two sub-cases could arise. The first one would be such that $\bar{\theta} \leq \frac{p_{A}-X}{q_{A}-q_{B}}$, implying that there is no user who would buy product $A$ if he has illegal access to product $B$. This, however, never occurs since in the equilibrium, developer $A$ sets the price low enough that users with $\theta$ close to $\bar{\theta}$ always prefer to buy the legal version of $A$ (see Appendix 3.4.1). The second situation appears when $\frac{p_{A}-X}{q_{A}-q_{B}}<\bar{\theta}$, implying that such users exist, and their number is higher than zero. So next, we discuss this only feasible sub-case.

Both competitors introduce physical protection and $\frac{p_{A}-X}{q_{A}-q_{B}}<\bar{\theta}$. In this case, there are users who prefer the legal version of the higher quality product $q_{A}$ even though they have 


$$
\begin{aligned}
& 1-c_{A} \ldots \text { illegal } \mathrm{A} \\
& 1-\mathrm{c}_{A} \ldots \text { illegal A } \quad\left(1-\mathrm{c}_{B}\right) \mathrm{c}_{A} \ldots \text { illegalB } \quad 1-\mathrm{c}_{A} \ldots \text { illegal A } \\
& \mathrm{c}_{A} \ldots \text { no prodyct } \mathrm{c}_{A} \ldots \text { illegal } \AA_{A} \mathrm{c}_{B} \ldots \mathrm{B} \quad 1-\mathrm{c}_{A} \ldots \text { illegal } \mathrm{q}_{4} \ldots \mathrm{A} \\
& \left(1-c_{B}\right) c_{A} \ldots \text { illegal B } \quad\left(1-c_{B}\right) c_{A} \ldots \text { illegal B } \\
& \begin{array}{lll}
\text { no product } & \mathrm{c}_{A} \mathrm{c}_{B} \ldots \text { no product } & \mathrm{c}_{A} \mathrm{c}_{B} \ldots \mathrm{A} \\
+\quad \mid+\frac{X}{q_{A}} \quad \theta=\frac{X}{q_{B}} \quad \theta=\frac{p_{B}}{q_{B}} \quad \theta=\frac{p_{A}-p_{B}}{q_{A}-q_{B}} \quad \theta=\frac{p_{A}-X}{q_{A}-q_{B}} \quad \bar{\theta}
\end{array}
\end{aligned}
$$

Figure 12: Both developers introduce protection, and $\frac{p_{A}-X}{q_{A}-q_{B}}<\bar{\theta}$

access to the illegal version of product $B$, but not of product $A$. This leads to the following market coverage:

From the distribution of users on the market, we obtain the following demand for the individual products:

$$
\begin{aligned}
D_{A} & =c_{A} c_{B}\left(\bar{\theta}-\frac{p_{A}-p_{B}}{q_{A}-q_{B}}\right)+c_{A}\left(1-c_{B}\right)\left(\bar{\theta}-\frac{p_{A}-X}{q_{A}-q_{B}}\right) \\
& =\frac{c_{A}\left(X\left(1-c_{B}\right)+\bar{\theta}\left(q_{A}-q_{B}\right)+c_{B} p_{B}-p_{A}\right)}{q_{A}-q_{B}}, \\
D_{B} & =c_{A} c_{B}\left(\frac{p_{A}-p_{B}}{q_{A}-q_{B}}-\frac{p_{B}}{q_{B}}\right) .
\end{aligned}
$$

As in the previous section, we start with determining the range of the expected penalty values $X$ such that this sub-case is a Nash equilibrium in prices. Recall that for the existence of a price equilibrium in the case when only developer $A$ adopts protection, $X$ has to be low enough from the perspective of developer $A$, but it has to be high enough from the view point of developer $B$. Now in the case under consideration, there are no such opposing requirements on $X$, since for both developers to charge high prices (above $X$ ), they both "need" $X$ to be low ${ }^{48}$. Intuitively, if $X$ is close to zero, then both developers would implement protection and charge prices above $X$ rather than adjust their prices to $X$ or below. We show in Appendix 3.4.4 that a strictly positive $\underline{X}<\underline{X}_{0}=\frac{\bar{\theta} q_{B}\left(q_{A}-q_{B}\right)}{4 q_{A}-q_{B}}$ (note that $\underline{X}_{0}$ equals

\footnotetext{
${ }^{48}$ Certainly, if the developers could costlessly choose $X$, they would set it sufficiently high to exclude illegal use, so "need" is used in the sense of pure mathematical conditions for an equilibrium in the given range. Also note that since these mathematical conditions for both developers stipulate an upper bound, the analysis is to some extent simpler than in the case of developer $A$ alone implementing protection as it is impossible that the intersection of conflicting requirements on $X$ results in an empty set.
} 
$p_{B}^{o}$ of the pure Bertrand equilibrium) exists such that the following prices constitute an equilibrium:

$$
\begin{aligned}
p_{A}^{*} & =2 q_{A} \frac{\bar{\theta}\left(q_{A}-q_{B}\right)+X\left(1-c_{B}\right)}{4 q_{A}-c_{B} q_{B}}, \\
p_{B}^{*} & =\frac{\bar{\theta}\left(q_{A}-q_{B}\right)+X\left(1-c_{B}\right)}{4 q_{A}-c_{B} q_{B}} q_{B} .
\end{aligned}
$$

As for a comparative statics analysis with respect to $c_{A}$ and $c_{B}$, it is straightforward to show that equilibrium prices do not depend on $c_{A}$ and increase in $c_{B}$. While the positive effect of $c_{B}$ is not unexpected, the independence of the equilibrium prices on $c_{A}$ might seem less intuitive. However, if both developers charge prices above $X$, any consumer not controlled by developer $A$ would use an illegal version of product $A$, and a small change in $c_{A}$ would only have a market size effect, i.e., both demands would change proportionally to the change in $c_{A}$. As there are no production costs, the change in marginal incentives will be also proportional to the change in $c_{A}$, so that the prices do not change. Note also that both developers prefer the good protection of a competitor's product, that is $\frac{\partial \Pi_{A}^{*}}{\partial c_{B}}>0$ and $\frac{\partial \Pi_{B}^{*}}{\partial c_{A}}>0$. The intuition is that an increase in either $c_{A}$ or $c_{B}$ increases the number of legal users for both developers, as it could be seen by visual inspection that $\frac{\partial D_{A}}{\partial c_{B}}>0$ and $\frac{\partial D_{B}}{\partial c_{A}}>0$ and also by looking at the market coverage in Figure 12 .

Before proceeding to the central issue of our analysis - the interaction between the private and public IPR protection -we make two additional assumptions: 1) $\left|\frac{\partial^{2} \pi_{i}^{*}}{\partial c_{i} \partial c_{i}}\right|>\left|\frac{\partial^{2} \pi_{i}^{*}}{\partial c_{i} \partial c_{j}}\right|$, and 2) $c_{B} \leq \frac{1}{2}$. Assumption 1) is a rather standard implying the uniqueness of the equilibrium values of $c_{A}^{*}$ and $c_{B}^{*}$ as well as its stability. As for assumption 2), we argue here that the most plausible optimal values of $c_{B}$ are in the range of $\left(0, \frac{1}{2}\right)$. The reason for this is rather a tough price competition in the vertically differentiated market. Consequently, the lower quality producer charges a substantially lower price and usually earns only a small fraction of the high-quality developer's profit in equilibrium. Thus, developer $B$ cannot afford to expand $c_{B}$ much above zero due to the increasing marginal cost of private protection (recall that $\left.\frac{\partial^{2} h}{\partial c_{i}^{2}}\left(c_{i}\right)>0\right)$.

Proposition 2 Let the protection cost function $h(c)$ be such that assumptions 1) and 2) above hold, then an increase in $X$ leads to an increase in the optimal protection of both developers, that is, $\frac{d c_{A}^{*}}{d X}>0$ and $\frac{d c_{B}^{*}}{d X}>0$. Thus, private and public IPR protections are strategic complements.

Proof. see Appendix 3.4.6 
The sign and the size of interaction between the public and private IPR protection, $\frac{d c_{i}^{*}}{d X}$, depends on the impact of the expected penalty, $X$, on the marginal profitability of both developers' private protection, or, more technically, on the signs of both $\frac{\partial^{2} \pi_{A}^{*}}{\partial c_{A} \partial X}$ and $\frac{\partial^{2} \pi_{B}^{*}}{\partial c_{B} \partial X}$. It turns out that $\frac{\partial^{2} \pi_{A}^{*}}{\partial c_{A} \partial X}>0$ for all permissible values, and $\frac{\partial^{2} \pi_{B}}{\partial c_{B} \partial X}>0$ for (at least) all values of $c_{B}$ such that $c_{B} \leq \frac{1}{2}$ (see Appendix 3.4.6).

So in the situation when the expected penalty is low (that is, $X \in(0, \underline{X}])$, there is strategic complementarity not only between the private and public protections but also between the two private protections that reinforce each other (recall that $\frac{\partial^{2} \pi_{i}^{*}}{\partial c_{A} \partial c_{B}}>0$ ). In this case, an increase in the private protection of one developer induces the increase in the optimal protection of the other developer. Thus, the "cost effect" is not dominant here unlike in the case when only the high-quality developer adopts protection (see section 4.3.1 ) because here an increase in $X$ leads to an increase of both $c_{A}$ and $c_{B}$ causing an upward spiral in private protections until the new equilibrium is reached.

As before, the nature of the interaction between private and public IPR is the key ingredient in analyzing the comparative statics effects of $X$ on equilibrium prices and profits.

Lemma 8 An increase in $X$ leads to a rise in both prices and profits for both developers.

Proof. Directly from equilibrium prices (21) and from profit comparison (in Mathematica file).

Note also that as both protections $c_{A}, c_{B}$ tend to perfect protections, the equilibrium prices and profits go to profit from pure Bertrand competition.

\subsection{Key section results}

In this section, we concentrated on how the change in expected penalty affects developers' equilibrium values when producers implement physical protection. Predictably, the initial size of the expected penalty plays the decisive role in shaping the behavior of the market participants. We concentrate on the cases where $X$ has an impact on the optimal protection $c^{*}$ at the margin.

Thus, if $X$ zero or small, then both developers introduce protection, and an increase in $X$ reinforces $c_{A}$ and $c_{B}$, that is, $\frac{d *_{A}}{d X}>0$ and $\frac{d c_{B}^{*}}{d X}>0$. This means the regulator's and developers' IPR are strategic complements. It is important to note that even for a zero or low expected punishment, it is never the case that all of the users that have access to the 
illegal versions would use only these illegal versions in equilibrium. (If this were the case, $X$ would have no impact on the users' and consequently on the the developers' decisions on either $c_{A}$ or $c_{B}$.) Thus, in an equilibrium with low $X$, some of the users with a high appreciation for quality who have illegal access to product $B$ would still buy legal versions of product $A$. An increase in $X$ would make product $A$ more attractive for those users. As an optimal response, developer $A$ would increase $c_{A}$ that would in turn lead to larger profit. At the same time an increase in $X$ would leave more room for developer $B$ to increase his prices and profit via an increase in $c_{B}$.

For some intermediate values of $X$, only $A$ introduces protection. Here, however, an increase in $X$ leads to a direct increase in competitor $B$ 's demand, and thus has a substantially larger impact on $B$ 's price and profit than on $A$ 's corresponding values. So it is optimal for $A$ to decrease $c^{*}$ as a response to an increase in $X$ harming competitor $B$ and improving $A$ 's profit by lowering his protection costs, $h(c)$. So, the regulator's and the developers' IPR are strategic substitutes, that is $\frac{d c *}{d X}<0$ and this case, as we showed, appears only for a large enough $c^{*}$.

When $X \geq p_{A}^{o}$, there is no need for protection by any developers, so the regulator's IPR protection is in a sense an effective full substitute for the private developers' IPR protection.

Finally, we omit the Stackelberg competition as it happens to produce no new insights than those of the Bertrand competition.

\section{Conclusion}

In this essay, we study the interaction between the two instances of IPR protection in a duopoly software market. The first instance is associated with the level of a government's or regulator's protection that comes in the form of an expected penalty for violating IPR. The second instance represents the private IPR protection at the level of the developer. The latter appears in two forms: i) a restriction of additional consumer services for the illegal users and ii) in the physical protection of software. While i) discourages illegal usage and makes it less attractive, ii) makes illegal usage harder. Thus, we examine the market equilibria with the above two forms of developer protection. Before that, we considered as a benchmark case the situation when developers do not use any form of IPR.

We show that the expected penalty may affect both the market coverage and the corresponding market equilibria in all considered set-ups. In the benchmark case, for instance, 
when the developers do not implement any protection and the level of the expected penalty is low enough, the expected penalty serves as a price regulation instrument putting the cap on the price. Furthermore, the low expected penalty may force one of the developers, mainly the one with the lower product quality, to leave the market and establish the second one as the monopolist. In the case of a high expected penalty, where no user has the incentive to use a product illegally, it does not play any role, no matter whether the developers use IPR protection or not.

In the case of medium and low levels of an expected penalty when developers implement some form of protection, the resulting effect of the expected penalty crucially depends on the framework under consideration.

Thus, if the protection based on restriction of services happens to be the developer's optimal choice, we show that the illegal users of the product may recruit themselves either from price sensitive users (the low-end of the market) or from the middle part of the market. In the latter case, the illegal users create a "buffer" between the two groups of legal users, the one with the highest valuation for quality and the other with the lowest preference for quality. In this case, a marginal price change of one developer does not affect the profit of the other developer and, moreover, the high-quailty developer generates the same profit as if he were a monopolist constrained only by the size of the expected penalty. In any case, when firms protect their IPR by means of service restrictions, the expected penalty has an impact on market conduct and the developer's IPR protection only if it exceeds or goes below a certain threshold.

In the case where the protection comes in the form of physical protection, however, the very marginal change in the expected penalty in general affects the developers' optimal choice. Furthermore, when there is an implementation of physical protection against copying, the expected penalty, depending on its size and on the particular set-up, can be either a complement or a substitute to the developers' IPR protection.

We did not explicitly compare the two forms of private protections nor was it the aim of our analysis. It is clear, however, that the decision whether to implement physical protection or protection based on service restrictions, depends on the respective profitability of these two forms that in turn depend on the cost of implementing physical protection, the respective levels of such protection, and the height of the expected penalty for illegal usage. In the case of restricting product services, the high-quality developer seems to target better the users with the highest sensitivity to quality. More specifically, implementing physical protection 
instead of implementing a service restriction (or decreasing prices to an expected penalty), leads to losing some of the high-end (quality sensitive) users since a fraction of these users have access to an illegal version. (Note that the users with the highest quality are usually the most important source of a developer's profit). Moreover, implementing physical protection involves direct costs unlike the two other options. Thus, it seems that the physical protection would be optimal only if a developer can relatively cheaply achieve a high fraction of users who could use the product only legally and, when at the same time, the expected penalty is low enough, and the protection via additional services is not very effective.

As for the possible extensions of our analysis, the normative considerations would seem to be the most natural ones. In other words, the optimal regulator's choice of IPR protection and its economic impacts would be an issue. This would, in turn, require putting "more structure" in our model and consequently specifying the regulator's objective function. Since in our context, it was suitable to think of the two foreign developers competing on a third host market, the simplest case would be that the host regulator maximizes the consumer surplus net of the costs of implementing a particular level of expected penalty. This would further mean that the regulator would prefer to induce the most competitive set-up by means of the expected penalty, given the costs of reaching a particular level of expected penalty (whereby the costs of reaching a particular level are convex, that is above proportionally increasing in it). However, in our set-up where the users have access to an illegal version of the product, the choice of an optimal expected penalty seems to be trivial; in order to maximize the consumer surplus, the regulator will simply set the expected penalty to zero (or to some minimal level if zero is not feasible due to, say, an international standard and requirements for a minimal IPR protection). Thus, the set-up in which one or both developers are the domestic ones would be surely more interesting to analyze.

Another interesting extension would be to allow for the explicit trade-off between the increased developer IPR protection and the decreasing functionality of the product and to study the social welfare consequences and policy implications of such a trade-off. 


\section{References}

Banerjee, D.S. (2003): "Software Piracy: A Strategic Analysis and Policy Instruments," International Journal of Industrial Organization, Vol. 21(1), pp. 97-127.

Belleflamme, P. (2001): "Oligopolistic Competition, IT Use for Software Differentiation and the Productivity Paradox," International Journal of Industrial Organization, Vol. 19, pp. $227-248$.

Belleflamme, P., and Peitz, M. (2010) "Digital Piracy: Theory," CESifo Working Paper Series, No. 3222.

Belleflamme, P., and Picard, P.M. (2007): "Piracy and Competition," Journal of Economics and Management Strategy, Vol. 16, pp. 351-383.

Boom, A. (2001): "On the Desirability of Compatibility with Software Selection," Journal of Industrial Economics, Vol. 49, pp. 85-96.

Bulow, J., Geanakoplos, J., and Klemperer, P. (1985): "Multimarket Oligopoly: Strategic Substitutes and Strategic Complements," Journal of Political Economy, Vol. 93, pp. 488511.

Choi, P., Bae, S.H., and Jun, J. (2010): "Digital Piracy and Firms' Strategic Interactions: The Effects of Public Copy Protection and DRM Similarity," Information Economics and Policy, Vol. 22, pp. 354-364.

Economides, N.(1992): "Competition and Integration among Complements and Network Market Structure," Journal of Industrial Economics, Vol. 40, pp. 105-123.

Economides, N. (1996): "The Economics of Networks," International Journal of Industrial Organization, Vol. 16, pp. 673-699.

Farrell, J., and Klemperer, P. (2006): "Coordination and Lock-In: Competition with Switching Costs and Network Effects," CPC UC Berkeley Working Paper, No. 179157.

Fudenberg, D., and Tirole, J. (1984): "The Fat Cat Effect, the Puppy Dog Ploy and the Lean and Hungry Look," American Economic Review, Vol. 74, pp. 361-368.

Jain, S. (2008): "Digital Piracy: A Competitive Analysis," Marketing Science, Vol. 27, pp. 610-626. 
King, S.P., and Lampe, R. (2003): "Network Externalities, Price Discrimination and Profitable Piracy," Information Economics and Policy, Vol. 15, pp. 271-290.

Kúnin, M. (2004): "Why do Software Manufacturers Tolerate Piracy in Transition and Less Developed Countries?" CERGE-EI Working Paper, No. 231.

Kúnin, M., and Žigić, K. (2006): "Strategic Trade Policy and Vertical Product Differentiation: Intra-industry Trade between Developed and Developing Countries," CERGE-EI Working Paper, No. 230.

Minnitti, A., and Vergari, C. (2010): "Turning Piracy into Profits: A Theoretical Investigation," Information Economics and Policy, Vol. 22(4), pp. 379-390.

Peitz, M. and Waelbroeck, P. (2006): "Piracy of Digital Product: A Critical Review of the Theoretical Literature," Information Economics and Policy, Vol. 21(4), pp. 449-476.

Quélin, B.V., Abdessemed, T., and Bonardi, J.P. (2001): "Standardization of Network Technologies: Market Processes or the Result of Inter-firm Co-operation," Journal of Economic Survey, Vol. 15, pp.543-569.

Scotchmer, S. (2004): Innovation and Incentives, The MIT Press.

Shaked, A., and Sutton, J. (1984): Natural Oligopolies and International Trade: An Introduction, Oxford University Press.

Shapiro, C. (2003): "Antitrust Limits to Patent Settlements," RAND Journal of Economics, Vol. 34, pp. 391-411.

Shy, O., and Thisse, J. (1999): "A Strategic Approach to Software Protection," Journal of Economics \& Management Strategy, Vol. 8(2), pp. 163-190.

Stavins, J. (1995): "Model Entry and Exit in a Differentiated-Product Industry: The Personal Computer Market," The Review of Economics and Statistics, Vol. 77, pp. 571584 .

Sutton, J. (1991): Sunk Cost and Market Structure, The MIT Press.

Takeyama, L.N. (2009): "Copyright Enforcement and Product Quality Signaling in Markets for Computer Software," Information Economics and Policy, Vol. 21(4), pp. 291-296. 
Tirole, J. (1988): The Theory of Industrial Organization, The MIT Press.

Werden, G.J. (2001): "Network Effects and Conditions of Entry: Lessons from the Microsoft Case," Antitrust Law, Vol. 69, pp. 87-111.

Žigić, K. (2000): "Strategic Trade Policy, Intellectual Property Rights Protection, and North-South Trade," Journal of Development Economics, 2000, Vol. 61(1), pp. 27-60.

Internet Sources: www.tomshardware.com, www.idg.com, www.zive.cz, www.ifpi.org, www.ria.com, www.pro-music.org. 


\section{APPENDIX}

\section{Basic Model}

\subsection{General notes for all appendices}

Most of the calculations in this paper were performed using Mathematica and other similar software. The Mathematica file is available upon request.

In almost all model situations here, profit functions are concave (quadratic, or, in singular cases, linear) in the respective choice variables, so that an interior solution is always a (local) maximum. In the remaining situations, profit functions are explicitly assumed concave in the main text. Thus, second-order conditions always hold in equilibrium, so they are omitted everywhere below.

\subsection{Indifferent users}

From the user utility function it follows that indifferent users are characterized by the following quality sensitivities. The notation $\theta_{Y Z}$, where $Y$ and $Z$ can be one of $\{0, A, B\}$ implies that the users with $\theta<\theta_{Y Z}$ strictly prefer $Y$ to $Z$, and the users with $\theta>\theta_{Y Z}$ strictly prefer $Z$ to $Y$. Then

$$
\theta_{0 A}=\frac{p_{A}}{q_{A}}, \theta_{0 B}=\frac{p_{B}}{q_{B}}, \theta_{B A}=\frac{p_{A}-p_{B}}{q_{A}-q_{B}} .
$$

For the situations wherein developer $B$ competes with either developer $A$ 's product priced at $X$ or the illegal version thereof, also priced at $X$, we use the threshold $\theta_{B P}=\frac{X-p_{B}}{q_{A}-q_{B}}$.

\subsection{Bertrand competition}

\subsubsection{Pure Bertrand competition}

Profit functions are $\pi_{A}=\left(\bar{\theta}-\theta_{B A}\right) p_{A}$, and $\pi_{B}=\left(\theta_{B A}-\theta_{0 B}\right) p_{B}$, and from F.O.C., it follows that

$$
p_{A}^{o}=2 \bar{\theta} q_{A} \frac{\left(q_{A}-q_{B}\right)}{4 q_{A}-q_{B}}, p_{B}^{o}=\bar{\theta} q_{B} \frac{\left(q_{A}-q_{B}\right)}{4 q_{A}-q_{B}},
$$

so that the equilibrium profits are

$$
\pi_{A}^{o}=4 \bar{\theta}^{2} q_{A}^{2} \frac{q_{A}-q_{B}}{\left(4 q_{A}-q_{B}\right)^{2}}, \pi_{B}^{o}=\bar{\theta}^{2} q_{A} q_{B} \frac{q_{A}-q_{B}}{\left(4 q_{A}-q_{B}\right)^{2}} .
$$




\subsubsection{Bertrand competition, where only developer $B$ makes profit}

The profit function of developer $B$ is $\pi_{B}=\left(\theta_{B P}-\theta_{0 B}\right) p_{B}$, so that

$$
p_{B}^{*}=\frac{q_{B}}{2 q_{A}} X, \pi_{B}^{*}=X^{2} \frac{q_{B}}{4 q_{A}\left(q_{A}-q_{B}\right)} .
$$

\subsubsection{Bertrand competition with binding price $p_{A}$ equal to $X$}

Developer $A$ is limited to setting the price $p_{A}^{*}=X$. Thus, the profit functions are $\pi_{A}=$ $\left(\bar{\theta}-\theta_{B P}\right) X$, and $\pi_{B}=\left(\theta_{B P}-\theta_{0 B}\right) p_{B}$, so that $p_{B}^{*}, \pi_{B}^{*}$ are the same as in $(24)$, and

$$
\pi_{A}^{*}=X \frac{2 \bar{\theta} q_{A}\left(q_{A}-q_{B}\right)-X\left(2 q_{A}-q_{B}\right)}{2 q_{A}\left(q_{A}-q_{B}\right)} .
$$

\subsection{Stackelberg competition in prices}

\subsubsection{Stackelberg competition in prices}

First assume that the condition $p_{A} \leq X$ is not binding. Then the profit functions are $\pi_{A}=\left(\bar{\theta}-\theta_{B A}\right) p_{A}$ and $\pi_{B}=\left(\theta_{B A}-\theta_{0 B}\right) p_{B}$, and developer $B$ 's reaction function is $p_{B}\left(p_{A}\right)=$ $p_{A} \frac{q_{B}}{2 q_{A}}$. Substituting this into $\pi_{A}$ and maximizing, we obtain

$$
p_{A}^{*}=\bar{\theta} q_{A} \frac{q_{A}-q_{B}}{2 q_{A}-q_{B}},
$$

so that

$$
p_{B}^{*}=\frac{\bar{\theta}}{2} \frac{q_{A}-q_{B}}{2 q_{A}-q_{B}} q_{B}, \pi_{A}^{*}=\bar{\theta} q_{A}^{2} \frac{q_{A}-q_{B}}{2\left(2 q_{A}-q_{B}\right)}, \pi_{B}^{*}=\bar{\theta} q_{A} q_{B}^{2} \frac{q_{A}-q_{B}}{4\left(2 q_{A}-q_{B}\right)^{2}} .
$$

Recall that if $p_{A} \leq X$ is binding, then the Stackelberg outcome coincides with the Bertrand outcome.

\section{Lower quality to illegal users}

\section{$2.1 \quad$ Indifferent users}

As usual, the notation $\theta_{Y Z}$, where $Y$ and $Z$ can be one of $\{0, A, B, I\}$ implies that the users with $\theta<\theta_{Y Z}$ strictly prefer $Y$ to $Z$, and the users with $\theta>\theta_{Y Z}$ strictly prefer $Z$ to $Y$. Throughout this appendix, "product $P$ " refers to the illegal version of product $A$.

As in the basic model, for thresholds not involving the illegal version of product $A$,

$$
\theta_{0 A}=\frac{p_{A}}{q_{A}}, \theta_{0 B}=\frac{p_{B}}{q_{B}}, \theta_{B A}=\frac{p_{A}-p_{B}}{q_{A}-q_{B}} .
$$


For thresholds involving product $P$ but not involving product $B$,

$$
\theta_{0 P}=\frac{X}{\alpha q_{A}}, \theta_{P A}=\frac{p_{A}-X}{q_{A}-\alpha q_{A}} .
$$

As for the threshold between $B$ and $P$, two cases have to be distinguished. First, the quality reduction to illegal users can be relatively low so that $P$ is still better than $B$, i.e., $q_{B}<\alpha q_{A}$. Second, the quality reduction to illegal users can be relatively high so that illegal $A$ becomes worse than $B$, i.e., $q_{B}>\alpha q_{A}$. (If $q_{B}=\alpha q_{A}$, then it is impossible that both $B$ and $P$ are in the market, and we concentrate on the cases where all three products are present.) In the first case, users with their sensitivity below the threshold use $B$ whereas those above use $P$, so we use notation $\theta_{B P}$. In the second case, the situation is the opposite so we use notation $\theta_{P B}$. These are equal to

$$
\theta_{B P}=\frac{X-p_{B}}{\alpha q_{A}-q_{B}}, \theta_{P B}=\frac{p_{B}-X}{q_{B}-\alpha q_{A}} .
$$

(Mathematically, these two are identical.)

\subsection{Monopoly}

The relevant thresholds are $\theta_{0 A}, \theta_{0 P}$, and $\theta_{P A}$. Two cases are possible. First, if $p_{A} \leq \frac{X}{\alpha}$, then $\theta_{P A} \leq \theta_{0 A} \leq \theta_{0 P}$ (equality holds everywhere or nowhere) so that $P$ is out of the market and users buy either $A$ or nothing. Second, if $p_{A}>\frac{X}{\alpha}$, then $\theta_{P A}>\theta_{0 A}>\theta_{0 P}$ so that both $P$ and $A$ are in the market as in Figure 6 .

The monopolist's profit can be shown to be unimodal, and three outcomes can be distinguished.

First, if $X \geq \frac{1}{2} \bar{\theta} \alpha q_{A}$, then the unconstrained monopoly price is such that the illegal product is ousted, so that

$$
p_{A}^{*}=\frac{\bar{\theta} q_{A}}{2}, \pi_{A}^{*}=\frac{\bar{\theta}^{2} q_{A}}{4} .
$$

Second, if $X<\bar{\theta} \alpha q_{A} \frac{(1-\alpha)}{2-\alpha}$, then both $A$ and $P$ are present so that

$$
p_{A}^{*}=\frac{X+\bar{\theta} q_{A}(1-\alpha)}{2}, \pi_{A}^{*}=\frac{1}{4} \frac{\left(X+q_{A} \bar{\theta}(1-\alpha)\right)^{2}}{q_{A}(1-\alpha)} .
$$

Third, if $\bar{\theta} \alpha q_{A} \frac{(1-\alpha)}{2-\alpha} \leq X<\frac{1}{2} \bar{\theta} \alpha q_{A}$, then while the monopolist has to lower the price due to the possibility of illegal use, this illegal use is still eliminated at the optimum, namely

$$
p_{A}^{*}=\frac{X}{\alpha}, \pi_{A}^{*}=\frac{X}{\alpha}\left(\bar{\theta}-\frac{X}{\alpha q_{A}}\right) .
$$




\subsection{Bertrand competition}

\subsubsection{Market structure}

The following user distributions across products are possible depending on the prices.

Remark 5 Unless the fixed costs are prohibitive, the developers can always choose their prices so that both legal products are in the market, so that we neglect the price combinations such that either $A$ or $B$ (or both) are out.

If $p_{A} \leq \frac{X}{\alpha}$, then $P$ is out of the market and the outcome is the same duopoly as in the basic model.

If $p_{A}>\frac{X}{\alpha}$, then $P$ can be in the market, and it is necessary to distinguish between the general cases of $q_{B}<\alpha q_{A}$ and $q_{B}>\alpha q_{A}$ (we neglect the equality as singular). Let

$$
p_{B}^{P}=\frac{X}{\alpha} \frac{q_{B}}{q_{A}}, p_{B}^{T}=\frac{X\left(q_{A}-q_{B}\right)-p_{A}\left(\alpha q_{A}-q_{B}\right)}{q_{A}-\alpha q_{A}},
$$

and note that $p_{B}^{T} \lesseqgtr p_{B}^{P}$ iff $q_{B} \lesseqgtr \alpha q_{A}$.

Case $q_{B}<\alpha q_{A}$ : In this case, if $p_{B} \leq p_{B}^{T}$, then $P$ is out of the market, and if $p_{B}^{T}<p_{B}<p_{B}^{P}$, then all three products are present and the market structure corresponds to Figure 7 , i.e., the relevant thresholds are $\theta_{0 B}, \theta_{B P}$, and $\theta_{P A}$.

Case $q_{B}>\alpha q_{A}$ : In this case, if $p_{B} \leq p_{B}^{P}$, then $P$ is out of the market, and if $p_{B}^{P}<p_{B}<p_{B}^{T}$, then all three products are present and the market structure corresponds to Figure 8 , i.e., the relevant thresholds are $\theta_{0 P}, \theta_{P B}$, and $\theta_{B A}$.

Remark 6 In this paper, we concentrate on the cases where all three products, both the legal ones and illegal $A$, are in the market. Thus, we only consider equilibria such that $p_{A}^{*}>\frac{X}{\alpha}$, and $p_{B}^{*}$ is strictly between $p_{B}^{T}$ and $p_{B}^{P}$.

\subsubsection{Case $q_{B}<\alpha q_{A}$}

The profit functions are $\pi_{A}=\left(\bar{\theta}-\frac{p_{A}-X}{q_{A}-\alpha q_{A}}\right) p_{A}$ and $\pi_{B}=\left(\frac{X-p_{B}}{\alpha q_{A}-q_{B}}-\frac{p_{B}}{q_{B}}\right) p_{B}$, so that

$$
\begin{aligned}
p_{A}^{*}=\frac{X+\bar{\theta} q_{A}(1-\alpha)}{2}, p_{B}^{*}=\frac{X q_{B}}{2 \alpha q_{A}} \\
\pi_{A}^{*}=\frac{1}{4} \frac{\left(\bar{\theta} q_{A}(1-\alpha)+X\right)^{2}}{q_{A}(1-\alpha)}, \pi_{B}^{*}=\frac{1}{4} X^{2} \frac{q_{B}}{\alpha q_{A}\left(\alpha q_{A}-q_{B}\right)} .
\end{aligned}
$$


The conditions $p_{A}^{*}>\frac{X}{\alpha}$ and $p_{B}^{T}<p_{B}^{*}<p_{B}^{P}$ hold iff $X>0$, and

$$
X<X_{\alpha 1}=\frac{\bar{\theta} \alpha q_{A}\left(\alpha q_{A}-q_{B}\right)(1-\alpha)}{(2-\alpha) \alpha q_{A}-q_{B}} .
$$

Both profits are decreasing in $\alpha$ when $0<X<X_{\alpha 1}$.

\subsubsection{Case $q_{B}>\alpha q_{A}$}

The profit functions of the developers are $\pi_{A}=\left(\bar{\theta}-\frac{p_{A}-p_{B}}{q_{A}-q_{B}}\right) p_{A}$, and $\pi_{B}=\left(\frac{p_{A}-p_{B}}{q_{A}-q_{B}}-\frac{p_{B}-X}{q_{B}-\alpha q_{A}}\right) p_{B}$, so that

$$
\begin{aligned}
p_{A}^{*} & =\frac{\left(q_{A}-q_{B}\right)\left(\bar{\theta} 2 q_{A}(1-\alpha)+X\right)}{\left(4 q_{A}-3 q_{A} \alpha-q_{B}\right)}, \\
p_{B}^{*} & =\left(q_{A}-q_{B}\right) \frac{\bar{\theta}\left(q_{B}-q_{A} \alpha\right)+2 X}{\left(4 q_{A}-3 q_{A} \alpha-q_{B}\right)} \\
\pi_{A}^{*} & =\left(q_{A}-q_{B}\right)\left(\frac{2 \bar{\theta} q_{A}(1-\alpha)+X}{4 q_{A}-3 q_{A} \alpha-q_{B}}\right)^{2}, \text { and } \\
\pi_{B}^{*} & =(1-\alpha) \frac{\left(q_{A}-q_{B}\right) q_{A}}{\left(q_{B}-\alpha q_{A}\right)}\left(\frac{\bar{\theta}\left(q_{B}-\alpha q_{A}\right)+2 X}{4 q_{A}-3 q_{A} \alpha-q_{B}}\right)^{2} .
\end{aligned}
$$

The conditions $p_{A}^{*}>\frac{X}{\alpha}$ and $p_{B}^{P}<p_{B}^{*}<p_{B}^{T}$ hold iff $X \geq 0$, and

$$
X<X_{\alpha 2}=\bar{\theta} \frac{\left(q_{A}-q_{B}\right)\left(q_{B}-q_{A} \alpha\right) \alpha q_{A}}{4 q_{A} q_{B}-q_{B}^{2}-2 \alpha q_{A}^{2}-\alpha q_{A} q_{B}} .
$$

Both profits are decreasing in $\alpha$ when $0 \leq X<X_{\alpha 2}$.

\subsection{Stackelberg competition}

The only relevant case is $q_{B}>\alpha q_{A}$. Developer $B$ 's reaction function is

$$
p_{B}\left(p_{A}\right)=\frac{\left(X q_{A}-X q_{B}+p_{A}\left(q_{B}-\alpha q_{A}\right)\right)}{2 q_{A}-2 \alpha q_{A}} .
$$

Substituting this into the profit function of developer $A$ and solving for $p_{A}$, we obtain

$$
p_{A}^{*}=\frac{1}{2} \frac{2 \bar{\theta} q_{A} \alpha-2 \bar{\theta} q_{A}-X}{\alpha q_{A}-2 q_{A}+q_{B}}\left(q_{A}-q_{B}\right),
$$


so that

$$
\begin{aligned}
p_{B}^{*} & =\frac{1}{2} \frac{\left(q_{A}-q_{B}\right)\left(X\left(q_{B}-4 q_{A}+3 \alpha q_{A}\right)+\bar{\theta} 2 q_{A}(\alpha-1)\left(q_{B}-\alpha q_{A}\right)\right)}{\left(2 q_{A}-2 \alpha q_{A}\right)\left(q_{B}-2 q_{A}+\alpha q_{A}\right)}, \\
\pi_{A}^{*} & =\frac{1}{8}\left(q_{A}-q_{B}\right) \frac{\left(2 \bar{\theta} q_{A}(1-\alpha)+X\right)^{2}}{\left(2 q_{A}-\alpha q_{A}-q_{B}\right) q_{A}(1-\alpha)}, \text { and } \\
\pi_{B}^{*} & =\frac{1}{16 q_{A}} \frac{\left(2 \bar{\theta} q_{A}\left(q_{B}-\alpha q_{A}\right)(1-\alpha)+X\left(4 q_{A}-3 q_{A} \alpha-q_{B}\right)\right)^{2}\left(q_{A}-q_{B}\right)}{\left(-q_{B}+\alpha q_{A}\right)(-1+\alpha)\left(-2 q_{A}+\alpha q_{A}+q_{B}\right)^{2}} .
\end{aligned}
$$

The conditions $p_{A}^{*}>\frac{X}{\alpha}$ and $p_{B}^{P}<p_{B}^{*}<p_{B}^{T}$ hold iff $X \geq 0$, and

$$
X<X_{\alpha S}=\bar{\theta} \frac{2\left(q_{A}-q_{B}\right)\left(q_{B}-q_{A} \alpha\right) \alpha(1-\alpha) q_{A}}{\left(8-7 \alpha+\alpha^{2}\right) q_{A} q_{B}-(4-3 \alpha)\left(q_{B}^{2}+\alpha q_{A}^{2}\right)} .
$$

Both profits are higher than under Bertrand competition and decrease in $\alpha$ when $0 \leq$ $X<X_{\alpha S}$.

\section{Developers implement physical protection}

\subsection{Indifferent users}

As usual, the notation $\theta_{Y Z}$, where $Y$ and $Z$ can be one of $\{0, A, P, B, I\}$ implies that the users with $\theta<\theta_{Y Z}$ strictly prefer $Y$ to $Z$, and the users with $\theta>\theta_{Y Z}$ strictly prefer $Z$ to $Y$. Throughout this appendix, "product $P$ " refers to the illegal version of product $A$, and "product $I$ " refers to the illegal version of product $B$.

As in the basic model, for thresholds not involving the illegal products,

$$
\theta_{0 A}=\frac{p_{A}}{q_{A}}, \theta_{0 B}=\frac{p_{B}}{q_{B}}, \theta_{B A}=\frac{p_{A}-p_{B}}{q_{A}-q_{B}} .
$$

For thresholds involving product $P$, note that all consumers prefer $P$ to $I$, and the decision between $P$ and $A$ is made on the basis of prices alone. The remaining thresholds are

$$
\theta_{0 P}=\frac{X}{q_{A}}, \theta_{B P}=\frac{X-p_{B}}{q_{A}-q_{B}}
$$

For thresholds involving product $I$, note that the decision between $I$ and $B$ is made on the basis of prices alone. The remaining thresholds are

$$
\theta_{0 I}=\frac{X}{q_{B}}, \theta_{I A}=\frac{p_{A}-X}{q_{A}-q_{B}} .
$$

Also recall that the illegal products are available only to the fractions of consumers not controlled by the corresponding firms. 


\subsubsection{The price-quality ratio rule}

The following general result can be easily shown to hold.

Lemma 9 If there is a good of quality $q_{A}$ available at price $p_{A}$ and a good of quality $q_{B}<q_{A}$ available at price $p_{B}$, then a necessary condition exists for consumers to buy good $B$, namely the price per unit of quality is strictly lower for the lower quality good, i.e., $\frac{p_{B}}{q_{B}}<\frac{p_{A}}{q_{A}}$.

Proof. The claim directly follows from $\theta_{B A}-\theta_{0 B}>0$.

This result was implicitly used in previous chapters, and the equilibrium prices complied with it. However, in this chapter, profit functions are not unimodal, and an analysis of deviations requires the Lemma above explicitly.

Corollary 2 No consumer with access to $P$ prefers $B$ to $P$ if $p_{B} \geq X \frac{q_{B}}{q_{A}}$.

Corollary 3 No consumer with access to I prefers I to $A$ if $p_{A} \leq X \frac{q_{A}}{q_{B}}$.

\subsection{Duopoly: general notes}

Recall that the physical protection settings imply that every consumer is controlled by firm $A$ with probability $c_{A}$, and independently by firm $B$ with probability $c_{B}$. Thus, four groups of consumers exist.(In all cases, it is assumed that $\bar{\theta}$ is high enough.)

1. Consumers controlled by both firms, $c_{A} c_{B}$ : These consumers view the market as a standard duopoly, so that the following applies according to the price-quality ratio rule.

(a) If $\frac{p_{B}}{q_{B}}<\frac{p_{A}}{q_{A}}$, then the consumers with $\theta<\theta_{0 B}$ use nothing, those with $\theta_{0 B}<\theta<$ $\theta_{B A}$ buy product $B$, and those with $\theta_{B A}<\theta<\bar{\theta}$ buy product $A$.

(b) If $\frac{p_{B}}{q_{B}} \geq \frac{p_{A}}{q_{A}}$, then the consumers with $\theta<\theta_{0 A}$ use nothing, and those with $\theta_{0 A}<$ $\theta<\bar{\theta}$ buy product $A$.

2. Consumers controlled by firm $A$ alone, $c_{A}\left(1-c_{B}\right)$ : If $p_{B} \leq X$, then product $I$ is irrelevant, and the outcome is a standard duopoly as in group 1 . If $p_{B}>X$, then these consumers choose between $A$ and $I$ so that the following applies.

(a) If $p_{A}>X \frac{q_{A}}{q_{B}}$, then the consumers with $\theta<\theta_{0 I}$ use nothing, those with $\theta_{0 I}<\theta<$ $\theta_{I A}$ use product $I$, and those with $\theta_{I A}<\theta<\bar{\theta}$ buy product $A$. 
(b) If $p_{A} \leq X \frac{q_{A}}{q_{B}}$, then the consumers with $\theta<\theta_{0 A}$ use nothing, and those with $\theta_{0 A}<\theta<\bar{\theta}$ buy product $A$.

3. Consumers controlled by firm $B$ alone, $\left(1-c_{A}\right) c_{B}$ : If $p_{A} \leq X$, then product $P$ is irrelevant, and the outcome is a standard duopoly as in group 1. If $p_{A}>X$, then these consumers choose between $P$ and $B$ so that the following applies.

(a) If $p_{B}<X \frac{q_{B}}{q_{A}}$, then the consumers with $\theta<\theta_{0 B}$ use nothing, those with $\theta_{0 B}<\theta<$ $\theta_{B P}$ buy product $B$, and those with $\theta_{B P}<\theta<\bar{\theta}$ use product $P$.

(b) If $p_{B} \geq X \frac{q_{B}}{q_{A}}$, then the consumers with $\theta<\theta_{0 P}$ use nothing, and those with $\theta_{0 P}<\theta<\bar{\theta}$ use product $P$.

4. Consumers controlled by neither firm, $\left(1-c_{A}\right)\left(1-c_{B}\right)$ : The outcome in this group is the same as in group 3 due to the price-quality ratio rule. Namely, all consumers not controlled by firm $A$ have access to a good of quality $q_{A}$ at a price of no more than $X$. Then no such consumer will be interested in a product of quality $q_{B}$ if offered at a price above $X \frac{q_{B}}{q_{A}}<X$, so it is irrelevant whether these consumers are controlled by firm $B$.

Thus, the last two groups can be united into a single group of those not controlled by $A$, with the total measure of $c_{A}$. Also note that if $p_{A} \leq X$, then the outcome is that of a standard duopoly as both illegal products are dominated by product $A$.

Note that in this model, the duopoly is always viable in the sense that the low-quality developer can always set a price such that the demand for $B$ is strictly positive, e.g., $p_{B}=$ $\frac{\min \left\{p_{A}, X\right\} q_{B}}{2 q_{A}}$. Therefore, situations such that developer $B$ is out of the market, e.g., $p_{B} \geq p_{A}$, can be neglected except in reaction functions.

From the above, it follows that every consumer depending on the firms controlling and the relative position of the prices w.r.t. $X$, faces one of the following three situations.

- Case I: a standard duopoly, the choice between $A$ at $p_{A}$ and $B$ at $p_{B}$.

- Case II: the choice between $P$ at $X$ and $B$ at $p_{B}$.

- Case III: the choice between $A$ at $p_{A}$ and $I$ at $X$. 
The correspondence between these three cases, the consumer groups, and price settings, is the following $\left(p_{B}<p_{A}\right.$ assumed).

$\begin{array}{llll} & p_{A} \leq X & p_{B} \leq X<p_{A} & X<p_{B} \\ c_{A} c_{B} & \mathrm{I} & \mathrm{I} & \mathrm{I} \\ c_{A}\left(1-c_{B}\right) & \mathrm{I} & \mathrm{I} & \mathrm{III} \\ 1-c_{A} & \mathrm{I} & \mathrm{II} & \mathrm{II}^{49}\end{array}$

The approach to equilibrium verification is the following. First, the reaction functions are investigated, where it is assumed that the other developer's price satisfies the given constraints, and then it is checked whether it is optimal for this developer to charge a price in the relevant range. Second, equilibrium prices are computed from the corresponding firstorder conditions, and constraints on parameters are finalized. This approach is necessary as the profit functions feature discontinuity and non-unimodality.

\subsection{Bertrand competition where only $A$ implements protection} $c_{A}=c$

As stated in Chapter 4, we are interested in the subcase $p_{B}<X \frac{q_{B}}{q_{A}}, X<p_{A}$.

\subsubsection{Reaction function of developer $A$}

Let $p_{B}<X \frac{q_{B}}{q_{A}}$. Then developer $A$ 's demand function is described by the following.

1. Case (D): If $X<p_{A} \leq p_{B}+\bar{\theta}\left(q_{A}-q_{B}\right)$, then the situation that we focus on in the main text takes place,

$$
D_{A}=c\left(\bar{\theta}-\theta_{B A}\right)
$$

2. Case (d): If $p_{B} \frac{q_{A}}{q_{B}}<p_{A} \leq X$, then the outcome is that of an unconstrained duopoly,

$$
D_{A}=\bar{\theta}-\theta_{B A}
$$

3. Case $(\mathrm{m})$ : If $p_{A} \leq p_{B} \frac{q_{A}}{q_{B}}$, then developer $A$ is unconstrained,

$$
D_{A}=\bar{\theta}-\theta_{0 A} .
$$

Given the range of $p_{B}$, this demand function is continuous between cases (d) and (m) but not at $p_{A}=X$ unless $c=1$. The resulting profit function $\pi_{A}=p_{A} D_{A}$ is unimodal between (d) and $(\mathrm{m})$, and is discontinuous at $p_{A}=X$. 
An interior solution in case (D) can occur only if

$$
X<X_{d}=\frac{\bar{\theta}\left(q_{A}-q_{B}\right) q_{A}}{2 q_{A}-q_{B}}
$$

(Note, however, that $X_{d}$ is always larger than the pure Bertrand duopoly price, that is $X_{d}>p_{A}^{o}=X_{c u}$.)

In this case, the reaction function and the corresponding profit are given by

$$
r_{A}\left(p_{B}\right)=\frac{\bar{\theta}\left(q_{A}-q_{B}\right)+p_{B}}{2}, \pi_{A}\left(p_{B}\right)=\frac{c\left(\bar{\theta}\left(q_{A}-q_{B}\right)+p_{B}\right)^{2}}{4\left(q_{A}-q_{B}\right)},
$$

and an interior solution in (D) implies here that the maximum outside (D) is reached at $p_{A}=X$. Therefore, the profit above has to be compared with the profit in case (d), which equals

$$
\pi_{A}^{d}=X\left(\bar{\theta}-\frac{X-p_{B}}{q_{A}-q_{B}}\right) .
$$

While it is possible to make a direct comparison between $\pi_{A}\left(p_{B}\right)$ and $\pi_{A}^{d}$ and obtain the conditions such that there is no deviation to $(\mathrm{m})$, the calculation of it would be rather cumbersome, so we postpone it to the equilibrium analysis. However, it is immediately clear that the protection duopoly profit is higher at $X=0$ unless $c=0$.

\subsubsection{Reaction function of developer $B$}

Let $X<p_{A}$. Then developer $B$ 's demand function is described by the following.

1. Case (X): If $X \frac{q_{B}}{q_{A}} \leq p_{B}<X$, then no user not controlled by $A$ buys $B$ as all such users prefer $P$,

$$
D_{B}=c\left(\theta_{B A}-\theta_{0 B}\right) \text {. }
$$

2. Case (D): If $p_{B}<X \frac{q_{B}}{q_{A}}$, then the situation that we focus on in the main text takes place,

$$
D_{B}=c\left(\theta_{B A}-\theta_{0 B}\right)+(1-c)\left(\theta_{B P}-\theta_{0 B}\right) .
$$

Strictly speaking, this analysis should include situation $p_{B}<p_{A}-\bar{\theta}\left(q_{A}-q_{B}\right)$, but in equilibrium $p_{A}<\bar{\theta}\left(q_{A}-q_{B}\right)$, so this can be neglected.

This demand function is continuous; however, the resulting profit function $\pi_{B}=p_{B} D_{B}$ is generally non-unimodal between $(\mathrm{X})$ and $(\mathrm{D})$. 
An interior solution in case (D) occurs if $p_{A}<\left(1+\frac{1}{c}\right) X$, in which case the reaction function and the corresponding profit are given by

$$
r_{B}\left(p_{A}\right)=\frac{q_{B}}{2 q_{A}}\left(c p_{A}+(1-c) X\right), \pi_{B}\left(p_{A}\right)=\frac{q_{B}\left(c p_{A}+(1-c) X\right)^{2}}{4 q_{A}\left(q_{A}-q_{B}\right)} .
$$

However, in $(\mathrm{X})$, where the reaction function is the pure Bertrand reaction function $r_{B}\left(p_{A}\right)=$ $\frac{q_{B}}{2 q_{A}} p_{A}$, the condition $X \frac{q_{B}}{q_{A}} \leq p_{B}<X$ means that an interior maximum occurs if $2 X<p_{A}<$ $2 \frac{q_{A}}{q_{B}} X$, so that $\pi_{B}$ is not unimodal around $p_{B}=X_{\frac{q_{B}}{q_{A}}}$ if $2 X<p_{A}<\left(1+\frac{1}{c}\right) X$. If the constraint $p_{B} \leq X$ is neglected, then the global maximum of $\pi_{B}$ is attained in (D) when $p_{A} \leq\left(1+\frac{1}{\sqrt{c}}\right) X$. Then it can be shown that if $\left(1+\frac{1}{\sqrt{c}}\right) X \leq 2 \frac{q_{A}}{q_{B}} X$, i.e., if $c \geq\left(\frac{q_{B}}{2 q_{A}-q_{B}}\right)^{2}$, then the condition $p_{A} \leq\left(1+\frac{1}{\sqrt{c}}\right) X$ for the global maximum in (D) is both necessary and sufficient. If $c<\left(\frac{q_{B}}{2 q_{A}-q_{B}}\right)^{2}$, then the global maximum occurs in (D) for $p_{A} \leq \bar{p}_{A}^{D}$, where $\left(1+\frac{1}{\sqrt{c}}\right) X<\bar{p}_{A}^{D}<\left(1+\frac{1}{c}\right) X$ and

$$
\pi_{B}\left(\bar{p}_{A}^{D}\right)=\pi_{B}^{X}\left(\bar{p}_{A}^{D}\right)=c X\left(\frac{\bar{p}_{A}^{D}-X}{q_{A}-q_{B}}-\frac{X}{q_{B}}\right)
$$

which is the profit from deviation to $p_{B}=X$.

\subsubsection{Equilibrium calculation}

Assuming that all conditions on the prices hold, the equilibrium prices and profits are the following.

$$
\begin{aligned}
p_{A}^{*} & =\frac{2 \bar{\theta} q_{A}\left(q_{A}-q_{B}\right)+X(1-c) q_{B}}{4 q_{A}-c q_{B}}, \\
p_{B}^{*} & =q_{B} \frac{2 X(1-c)+\bar{\theta} c\left(q_{A}-q_{B}\right)}{4 q_{A}-c q_{B}}, \\
\pi_{A}^{*} & =c \frac{\left(2 \bar{\theta} q_{A}\left(q_{A}-q_{B}\right)+q_{B} X(1-c)\right)^{2}}{\left(4 q_{A}-q_{B} c\right)^{2}\left(q_{A}-q_{B}\right)}, \text { and } \\
\pi_{B}^{*} & =q_{A} q_{B} \frac{\left(2 X(1-c)+\bar{\theta} c\left(q_{A}-q_{B}\right)\right)^{2}}{\left(4 q_{A}-q_{B} c\right)^{2}\left(q_{A}-q_{B}\right)} .
\end{aligned}
$$

\subsubsection{Derivation of bounds on $X$ and $c$}

All conditions for these prices and profits to be interior local maxima are met if

$$
c \frac{\bar{\theta} q_{A}\left(q_{A}-q_{B}\right)}{2(1+c) q_{A}-c q_{B}}=X_{c l}<X<X_{c u}=2 \frac{\bar{\theta} q_{A}\left(q_{A}-q_{B}\right)}{4 q_{A}-q_{B}}
$$


where $X<X_{c u}$ follows from $p_{A}^{*}>X$, and $X>X_{c l}$ follows from $p_{B}^{*}<X \frac{q_{B}}{q_{A}}$, with the latter equivalent to $p_{A}^{*}<X\left(1+\frac{1}{c}\right)$. (Note that $X_{c l}<X_{c u}$.) It remains to be checked whether these maxima are global, i.e., that no developer prefers switching to a price corresponding to another market structure.

Developer $A$ can be shown not to switch to $p_{A}=X$ given $p_{B}=p_{B}^{*}$ if

$$
X \leq X_{c}^{+}=\frac{2 \bar{\theta} q_{A}\left(q_{A}-q_{B}\right)\left(4 q_{A}-c(2-c) q_{B}-\sqrt{1-c}\left(4 q_{A}-c q_{B}\right)\right)}{16 q_{A}^{2}-8 q_{A} q_{B}+\left(3 c-3 c^{2}+c^{3}\right) q_{B}^{2}},
$$

which is smaller than $X_{c u}$ when $c<1$. It turns out that $X_{c l} \lesseqgtr X_{c}^{+}$iff $c \gtreqless \frac{\sqrt{5}-1}{2} \approx 0.618034$, i.e., the (sub)case in question cannot occur if $c \leq \frac{\sqrt{5}-1}{2}$.

As for developer $B$, cases $c \geq\left(\frac{q_{B}}{2 q_{A}-q_{B}}\right)^{2}$ and $c<\left(\frac{q_{B}}{2 q_{A}-q_{B}}\right)^{2}$ are distinguished. In the former case, the condition to check is $p_{A}^{*} \leq X\left(1+\frac{1}{\sqrt{c}}\right)$, which is equivalent to

$$
X \geq X_{c}^{-}=2 \frac{\sqrt{c} \bar{\theta} q_{A}\left(q_{A}-q_{B}\right)}{(1+\sqrt{c})\left(4 q_{A}-\sqrt{c} q_{B}\right)},
$$

which is bigger than $X_{c l}$ when $c<1$. It can be shown that $X_{c}^{-} \lesseqgtr X_{c}^{+}$iff $c \gtreqless \underline{c}$, where

$$
\underline{c}=\frac{1}{3}\left(4-8(6 \sqrt{33}-26)^{-1 / 3}+(6 \sqrt{33}-26)^{1 / 3}\right) \approx 0.704402,
$$

so the lower bound on $c$ can be improved to $\underline{c}$ when $c \geq\left(\frac{q_{B}}{2 q_{A}-q_{B}}\right)^{2}$. In the other case, $c<\left(\frac{q_{B}}{2 q_{A}-q_{B}}\right)^{2}$, a direct comparison between $\pi_{B}^{*}$ and $\pi_{B}^{X}\left(p_{A}^{*}\right)$ yields a lower bound on $X$ located between $X_{c l}$ and $X_{c}^{-}$, which translates into a lower bound on $c$ located between $\frac{\sqrt{5}-1}{2}$ and $\underline{c}$. Note that given the lower bounds on $c$, case $c \geq\left(\frac{q_{B}}{2 q_{A}-q_{B}}\right)^{2}$ occurs with certainty if $\frac{q_{B}}{q_{A}}$ is not too high, namely, if $\frac{q_{B}}{q_{A}} \leq \approx 0.912622$.

\subsubsection{The effect of $X$ on $c$}

By the implicit function theorem,

$$
\frac{d c}{d X}=-\frac{\frac{\partial^{2} \Pi_{A}}{\partial c \partial X}}{\frac{\partial^{2} \Pi_{A}}{\partial c \partial c}},
$$

so that the sign of $\frac{\partial c}{\partial X}$ is the same as the sign of:

$$
\frac{\partial^{2} \Pi_{A}^{*}}{\partial c \partial X}=2 q_{B} \frac{2 \bar{\theta} q_{A}\left(q_{A}-q_{B}\right)\left(4 q_{A}+c q_{B}-8 c q_{A}\right)+X q_{B}(1-c)\left((4-12 c) q_{A}+\left(c+c^{2}\right) q_{B}\right)}{\left(q_{A}-q_{B}\right)\left(4 q_{A}-c q_{B}\right)^{3}} .
$$

The sign of this expression depends on the sign of $\left(4 q_{A}+c q_{B}-8 c q_{A}\right)$ and $\left((4-12 c) q_{A}+\left(c+c^{2}\right) q_{B}\right)$.

As $q_{B}<q_{A}$, both of these expressions can be shown to be negative for $c \geq \frac{4}{7} \approx 0.571429$.

Since it is shown above that the subcase in question can occur only if $c \geq \frac{\sqrt{5}-1}{2}>\frac{4}{7}$, both $\frac{\partial^{2} \Pi_{A}^{*}}{\partial c \partial X}$ and $\frac{d c}{d X}$ are negative. 


\subsubsection{The impact of $X$ on prices and profits}

First observe that $\frac{d \Pi_{A}^{*}}{d X}$ is clearly positive since $\frac{\partial \Pi_{A}^{*}}{\partial c}=0$ at the point of optimum. Thus,

$$
\frac{d \Pi_{A}^{*}}{d X}=\frac{\partial \Pi_{A}^{*}}{\partial c} \frac{d c}{d X}+\frac{\partial \Pi_{A}^{*}}{\partial X}=\frac{\partial \Pi_{A}^{*}}{\partial X}>0
$$

In the case of developer $B$, the impact of $X$ on developer $B$ 's profit is

$$
\frac{d \Pi_{B}^{*}}{d X}=\frac{\partial \Pi_{B}^{*}}{\partial c} \frac{d c}{d X}+\frac{\partial \Pi_{B}^{*}}{\partial X} .
$$

Since the indirect effect is negative and the direct one is positive, it cannot be told a priori which effect dominates. The same applies to both equilibrium prices.

\subsection{Bertrand competition where both developers implement pro- tection}

As stated in Chapter 4 , this case occurs if $X<p_{B}<p_{A}$.

\subsubsection{The non-existence of subcase $p_{A} \geq X+\bar{\theta}\left(q_{A}-q_{B}\right)$}

In this subcase, only the users controlled by both developers buy any legal products, so that the demands for the products are constant multiples of the standard duopoly demands, $D_{A}=c_{A} c_{B}\left(\bar{\theta}-\theta_{B A}\right)$ and $D_{B}=c_{A} c_{B}\left(\theta_{B A}-\theta_{0 B}\right)$. Therefore, if the solution is interior, then the equilibrium prices are identical to the standard duopoly equilibrium prices. In particular,

$$
p_{A}^{*}=2 \bar{\theta} q_{A} \frac{q_{A}-q_{B}}{4 q_{A}-q_{B}}<\bar{\theta}\left(q_{A}-q_{B}\right) \leq X+\bar{\theta}\left(q_{A}-q_{B}\right),
$$

which is a contradiction. Hence, the solution must be corner with $\frac{\partial \pi_{A}}{\partial p_{A}}<0$ at $p_{A}=X+\bar{\theta}\left(q_{A}-\right.$ $\left.q_{B}\right)+0$. However, it can be shown that this implies $\frac{\partial \pi_{A}}{\partial p_{A}}<0$ at $p_{A}=X+\bar{\theta}\left(q_{A}-q_{B}\right)-0$ as well (see the analysis of the profit and reaction functions below), so that $p_{A} \geq X+\bar{\theta}\left(q_{A}-q_{B}\right)$ is never optimal.

\subsubsection{The reaction function of developer $A$}

Let $X<p_{B}<\frac{q_{B}}{q_{A}}\left(X+\bar{\theta}\left(q_{A}-q_{B}\right)\right)$. (The upper limit on $p_{B}$ here follows from $p_{A}<$ $X+\bar{\theta}\left(q_{A}-q_{B}\right)$ and the price-quality ratio rule.) Then developer $A$ 's demand function is described by the following. 
1. Case (d): If $p_{A} \geq X+\bar{\theta}\left(q_{A}-q_{B}\right)$, then all users of product $A$ are completely controlled,

$$
D_{A}=c_{A} c_{B}\left(\bar{\theta}-\theta_{B A}\right) .
$$

2. Case (D): If $p_{B} \frac{q_{A}}{q_{B}}<p_{A}<X+\bar{\theta}\left(q_{A}-q_{B}\right)$, then the situation that we focus on in the main text takes place,

$$
D_{A}=c_{A} c_{B}\left(\bar{\theta}-\theta_{B A}\right)+c_{A}\left(1-c_{B}\right)\left(\bar{\theta}-\theta_{I A}\right) .
$$

3. Case (I): If $X \frac{q_{A}}{q_{B}}<p_{A} \leq p_{B} \frac{q_{A}}{q_{B}}$, then no one uses $B$,

$$
D_{A}=c_{A} c_{B}\left(\bar{\theta}-\theta_{0 A}\right)+c_{A}\left(1-c_{B}\right)\left(\bar{\theta}-\theta_{I A}\right) .
$$

4. Case (M): If $X<p_{A} \leq X \frac{q_{A}}{q_{B}}$, then no one uses $B$ or $I$,

$$
D_{A}=c_{A}\left(\bar{\theta}-\theta_{0 A}\right)
$$

5. Case (m): if $X \geq p_{A}$, then developer $A$ is unconstrained,

$$
D_{A}=\left(\bar{\theta}-\theta_{0 A}\right)
$$

Given the range of $p_{B}$, this demand function is continuous between cases (d) and (M) but not at $p_{A}=X$ unless $c_{A}=1$. The resulting profit function $\pi_{A}=p_{A} D_{A}$ is strictly decreasing in $p_{A}$ in $(\mathrm{d})$, unimodal between $(\mathrm{d})$ and $(\mathrm{M})$, and is discontinuous at $p_{A}=X$.

Denote $X^{A}=X\left(1-c_{B}\right)+\bar{\theta}\left(q_{A}-q_{B}\right)$. For cases (d), (D), (I), and (M), an interior solution in case (D) can occur only if

$$
X<X_{D}=\frac{\bar{\theta}\left(q_{A}-q_{B}\right) q_{B}}{2 q_{A}-q_{B}}, X<p_{B}<p_{B}^{D}=\frac{q_{B}}{2 q_{A}-c_{B} q_{B}} X^{A} .
$$

In this case, the reaction function and the corresponding profit are given by

$$
r_{A}\left(p_{B}\right)=\frac{X^{A}+c_{B} p_{B}}{2}, \pi_{A}\left(p_{B}\right)=\frac{c_{A}\left(X^{A}+c_{B} p_{B}\right)^{2}}{4\left(q_{A}-q_{B}\right)} .
$$

Now these values have to be compared with the monopoly profit in case $(\mathrm{m})$. Since $X<X_{D}$ implies $X<\frac{\bar{\theta} q_{B}}{2}$ in the relevant case, the monopoly profit is maximized at the highest $p_{A}$ in the range, i.e.,

$$
\pi_{A}^{m}=X\left(\bar{\theta}-\frac{X}{q_{A}}\right)
$$

While it is possible to make a direct comparison between $\pi_{A}\left(p_{B}\right)$ and $\pi_{A}^{m}$ and obtain the maximal value $\bar{X}\left(p_{B}\right)$ such that there is no deviation to $(\mathrm{m})$, the result is rather cumbersome. However, it is immediately clear that the duopoly profit is higher at $X=0$. 


\subsubsection{The reaction function of developer $B$}

Let $X \frac{q_{A}}{q_{B}}<p_{A}<X+\bar{\theta}\left(q_{A}-q_{B}\right)$. Then developer $B$ 's demand function is described by the following.

1. Case (D): If $X<p_{B}<p_{A} \frac{q_{B}}{q_{A}}$, then the situation that we focus on in the main text takes place,

$$
D_{B}=c_{A} c_{B}\left(\theta_{B A}-\theta_{0 B}\right)
$$

2. Case (X): If $X \frac{q_{B}}{q_{A}} \leq p_{B} \leq X$, then no one uses $I$,

$$
D_{B}=c_{A}\left(\theta_{B A}-\theta_{0 B}\right)
$$

3. Case (x): If $p_{B}<X \frac{q_{B}}{q_{A}}$, then there are consumers who prefer $B$ to $P$ (cf. the case when only $A$ implements protection),

$$
D_{B}=c_{A}\left(\theta_{B A}-\theta_{0 B}\right)+\left(1-c_{A}\right)\left(\theta_{B P}-\theta_{0 B}\right) .
$$

Strictly speaking, this analysis should include situations $p_{B}<p_{A}-\bar{\theta}\left(q_{A}-q_{B}\right)$ and even $p_{B}<X-\bar{\theta}\left(q_{A}-q_{B}\right)$, but in equilibrium $X<p_{A}<\bar{\theta}\left(q_{A}-q_{B}\right)$, so these can be neglected.

This demand function is continuous between cases $(\mathrm{X})$ and $(\mathrm{x})$ but not at $p_{B}=X$ unless $c_{B}=1$. The resulting profit function $\pi_{B}=p_{B} D_{B}$ is discontinuous at $p_{B}=X$ and can be non-unimodal between $(\mathrm{X})$ and $(\mathrm{x})$.

An interior solution in case (D) can occur only if $X<X_{D}$ (same as for developer $A$ ), in which case the reaction function and the corresponding profit have the same form as under a standard duopoly and are given by

$$
r_{B}\left(p_{A}\right)=\frac{q_{B}}{q_{A}} \frac{p_{A}}{2}, \pi_{B}\left(p_{A}\right)=c_{A} c_{B} \frac{p_{A}^{2} q_{B}}{4 q_{A}\left(q_{A}-q_{B}\right)} .
$$

If the maximum in (D) is interior, then the maximum in $(\mathrm{X})$ must be corner and the profit in $(\mathrm{X})$ is maximized at $p_{B}=X$, i.e.,

$$
\pi_{B}^{X}=c_{A} X\left(\frac{p_{A} q_{B}-X q_{A}}{\left(q_{A}-q_{B}\right) q_{B}}\right)
$$

As for $(\mathrm{x})$, the maximum is interior there if $p_{A}<X\left(1+\frac{1}{c_{A}}\right)$, then $\pi_{B}^{x}=\frac{q_{B}\left(c_{A}\left(p_{A}-X\right)+X\right)^{2}}{4 q_{A}\left(q_{A}-q_{B}\right)}$. It can be shown that if $p_{A}<X\left(1+\frac{1}{c_{A}}\right)$ and $c_{A}>c_{B}$, then deviation to (x) from (D) is always profitable (note that deviation to $(\mathrm{X})$ can be even more profitable). If $p_{A} \geq X\left(1+\frac{1}{c_{A}}\right)$, then $\pi_{B}$ strictly increases in $p_{B}$ in $(\mathrm{x})$, so that the maximal deviation profit is $\pi_{B}^{X}$ above. 


\subsubsection{Equilibrium calculation}

Assuming that all conditions on the prices hold, the equilibrium prices and profits are the following.

$$
\begin{aligned}
p_{A}^{*} & =2 q_{A} \frac{\bar{\theta}\left(q_{A}-q_{B}\right)+X\left(1-c_{B}\right)}{4 q_{A}-c_{B} q_{B}}, \\
p_{B}^{*} & =\frac{\bar{\theta}\left(q_{A}-q_{B}\right)+X\left(1-c_{B}\right)}{4 q_{A}-c_{B} q_{B}} q_{B}, \\
\pi_{A}^{*} & =4 c_{A} q_{A}^{2} \frac{\left(\bar{\theta}\left(q_{A}-q_{B}\right)+X\left(1-c_{B}\right)\right)^{2}}{\left(4 q_{A}-q_{B} c_{B}\right)^{2}\left(q_{A}-q_{B}\right)}, \text { and } \\
\pi_{B}^{*} & =c_{A} c_{B} q_{A} q_{B} \frac{\left(\bar{\theta}\left(q_{A}-q_{B}\right)+X\left(1-c_{B}\right)\right)^{2}}{\left(4 q_{A}-q_{B} c_{B}\right)^{2}\left(q_{A}-q_{B}\right)} .
\end{aligned}
$$

All condition for these prices and profits to be interior local maxima are met if

$$
X<\underline{X}_{0}=\frac{\bar{\theta} q_{B}\left(q_{A}-q_{B}\right)}{4 q_{A}-q_{B}} .
$$

It remains to check whether these maxima are global, i.e., that no developer prefers switching to a price corresponding to another market structure. As developer $B$ will always switch to a price below $X \frac{q_{B}}{q_{A}}$ if $p_{A}<X\left(1+\frac{1}{c_{A}}\right)$ and $c_{A}>c_{B}$, a necessary condition for no such deviation at $p_{A}=p_{A}^{*}$ is

$$
X<\frac{2 c_{A} q_{A}\left(q_{A}-q_{B}\right) \bar{\theta}}{2\left(2+c_{A}+c_{A} c_{B}\right) q_{A}-\left(1+c_{A}\right) c_{B} q_{B}},
$$

which is below $\underline{X}_{0}$ when $c_{A}<\frac{q_{B}}{2 q_{A}-q_{B}}$.

As for deviations to $p=X$ by either developer, let $\delta_{A}(X)=\pi_{A}^{*}(X)-\pi_{A}^{m}(X)$ and $\delta_{B}(X)=\pi_{B}^{*}(X)-\pi_{B}^{X}(X)$ be the differences between the duopoly and deviation profits. The functions $\delta_{i}(X)$ are positive at $X=0$ and decreasing in $X$ for $0<X<\underline{X}_{0}$. If $c_{A}$ is high enough, then it is possible that developer $A$ does not switch for all applicable $X$; however, developer $B$ always switches at $X=\underline{X}_{0}$, i.e. $\delta_{B}\left(\underline{X}_{0}\right)<0$. From this, it follows that $\exists \underline{X}$, $0<\underline{X}<\underline{X}_{0}$, such that the prices and profits above form an equilibrium.

\subsubsection{The effect of protection on prices and profits}

From the expressions for the equilibrium prices and profits, it is immediately seen that $c_{A}$ has no effect on prices. By algebraic derivation it can be shown that if $X<\underline{X}_{0}$ (and recall that the actual boundary is $\underline{X}<\underline{X}_{0}$ ), then both equilibrium prices and the net profit $\Pi_{A}^{*}=\pi_{A}^{*}-h\left(c_{A}\right)$ increase in $c_{B}$, and that the net profit $\Pi_{B}^{*}$ increases in $c_{A}$. 


\subsubsection{The effect of $X$ on $c_{A}$ and $c_{B}$}

Applying the implicit function theorem, we obtain:

$$
\begin{aligned}
& \frac{\partial \Pi_{A}^{*}}{\partial c_{A}}\left(c_{A}(X), c_{B}(X), X\right) \equiv 0 \Longrightarrow \frac{\partial^{2} \Pi_{A}^{*}}{\partial c_{A} \partial c_{A}} \frac{d c_{A}}{d X}+\frac{\partial^{2} \Pi_{A}^{*}}{\partial c_{A} \partial c_{B}} \frac{d c_{B}}{d X}+\frac{\partial^{2} \Pi_{A}^{*}}{\partial c_{A} \partial X} \equiv 0, \\
& \frac{\partial \Pi_{B}^{*}}{\partial c_{B}}\left(c_{A}(X), c_{B}(X), X\right) \equiv 0 \Longrightarrow \frac{\partial^{2} \Pi_{B}^{*}}{\partial c_{B} \partial c_{A}} \frac{d c_{A}}{d X}+\frac{\partial^{2} \Pi_{B}^{*}}{\partial c_{B} \partial c_{B}} \frac{d c_{B}}{d X}+\frac{\partial^{2} \Pi_{B}^{*}}{\partial c_{B} \partial X} \equiv 0 ;
\end{aligned}
$$

or, in matrix form:

$$
\left(\begin{array}{cc}
\frac{\partial^{2} \Pi_{A}^{*}}{\partial c_{A} \partial c_{A}} & \frac{\partial^{2} \Pi_{A}^{*}}{\partial c_{A} c_{B}} \\
\frac{\partial^{2} \Pi_{B}^{*}}{\partial c_{B} \partial c_{A}} & \frac{\partial^{2} \Pi_{B}^{*}}{\partial c_{B} \partial c_{B}}
\end{array}\right)\left(\begin{array}{c}
\frac{d c_{A}}{d X} \\
\frac{d c_{B}}{d X}
\end{array}\right)=\left(\begin{array}{c}
-\frac{\partial^{2} \Pi_{A}^{*}}{\partial c_{A} \partial X} \\
-\frac{\partial^{2} \Pi_{B}^{*}}{\partial c_{B} \partial X}
\end{array}\right)
$$

For simplicity, denote the first matrix as $H$; thus, $H=\left(\begin{array}{cc}\frac{\partial^{2} \Pi_{A}^{*}}{\partial c_{A} \partial c_{A}} & \frac{\partial^{2} \Pi_{A}^{*}}{\partial c_{A} \partial c_{B}} \\ \frac{\partial^{2} \Pi_{B}^{*}}{\partial c_{B} \partial c_{A}} & \frac{\partial^{2} \Pi_{B}^{*}}{\partial c_{B} \partial c_{B}}\end{array}\right)$. Applying Cramer's rule:

$$
\begin{aligned}
& \frac{d c_{A}}{d X}=\frac{\left|H_{A}\right|}{|H|}=\frac{1}{|H|}\left|\begin{array}{cc}
-\frac{\partial^{2} \Pi_{A}^{*}}{\partial c_{A} \partial X} & \frac{\partial^{2} \Pi_{A}^{*}}{\partial c_{A} \partial c_{B}} \\
-\frac{\partial^{2} \Pi_{B}^{*}}{\partial c_{B} \partial X} & \frac{\partial^{2} \Pi_{B}^{*}}{\partial c_{B} \partial c_{B}}
\end{array}\right|, \\
& \frac{d c_{B}}{d X}=\frac{\left|H_{B}\right|}{|H|}=\frac{1}{|H|}\left|\begin{array}{cc}
\frac{\partial^{2} \Pi_{A}^{*}}{\partial c_{A} \partial c_{A}} & -\frac{\partial^{2} \Pi_{A}^{*}}{\partial c_{A} \partial X} \\
\frac{\partial^{2} \Pi_{B}^{*}}{\partial c_{B} \partial c_{A}} & -\frac{\partial^{2} \Pi_{B}^{*}}{\partial c_{B} \partial X}
\end{array}\right| .
\end{aligned}
$$

Differentiating the equilibrium profits yields $\frac{\partial^{2} \Pi_{A}^{*}}{\partial c_{A} \partial c_{A}}=-h^{\prime \prime}\left(c_{A}\right)<0, \frac{\partial^{2} \Pi_{A}^{*}}{\partial c_{A} \partial X}>0$, and $\frac{\partial^{2} \Pi_{A}^{*}}{\partial c_{A} \partial c_{B}}>$ 0 for $X<\underline{X}_{0}$, and by our assumptions $\frac{\partial^{2} \Pi_{B}^{*}}{\partial c_{B} \partial c_{B}}<0$ as well. As for $\frac{\partial^{2} \Pi_{B}^{*}}{\partial c_{B} \partial c_{A}}=$

$q_{A} q_{B}\left(\bar{\theta}\left(q_{A}-q_{B}\right)+X\left(1-c_{B}\right)\right) \frac{\bar{\theta}\left(q_{A}-q_{B}\right)\left(4 q_{A}+q_{B} c_{B}\right)-X\left(12 c_{B} q_{A}-c_{B}^{2} q_{B}-4 q_{A}-q_{B} c_{B}\right)}{\left(4 q_{A}-q_{B} c_{B}\right)^{3}\left(q_{A}-q_{B}\right)}$,

which looks ambiguous, note that $\frac{\partial^{2} \Pi_{B}^{*}}{\partial c_{B} \partial c_{A}}=\frac{\partial^{2} \pi_{B}^{*}}{\partial c_{B} \partial c_{A}}$, and $\frac{\partial^{2} \pi_{B}^{*}}{\partial c_{B} \partial c_{A}}=\frac{1}{c_{A}} \frac{\partial \pi_{B}^{*}}{\partial c_{B}}$; then, F.O.C. $\frac{\partial \Pi_{B}^{*}}{\partial c_{B}}=0$ implies $\frac{\partial \pi_{B}^{*}}{\partial c_{B}}=h^{\prime}\left(c_{B}\right)$, so that $\frac{\partial^{2} \Pi_{B}^{*}}{\partial c_{B} \partial c_{A}}>0$. Finally, for $\frac{\partial^{2} \Pi_{B}^{*}}{\partial c_{B} \partial X}$,

$=-2 q_{B} q_{A} c_{A} \frac{\bar{\theta}\left(q_{A}-q_{B}\right)\left(4 q_{A}\left(2 c_{B}-1\right)-q_{B} c_{B}\right)+\left(1-c_{B}\right) X\left(4 q_{A}\left(3 c_{B}-1\right)-q_{B} c_{B}\left(1+c_{B}\right)\right)}{\left(4 q_{A}-q_{B} c_{B}\right)^{3}\left(q_{A}-q_{B}\right)}$,

it can be shown that for $X<\underline{X}_{0}$ and $c_{B} \leq 1 / 2, \frac{\partial^{2} \Pi_{B}^{*}}{\partial c_{B} \partial X}>0$. While the condition $c_{B} \leq 1 / 2$ cannot be loosened, this is a typical situation that we expect to occur in equilibrium, in which clearly $c_{B}^{*}<c_{A}^{*}$. Thus, we postulate $c_{B}^{*}<1 / 2$ so that $\frac{\partial^{2} \Pi_{B}^{*}}{\partial c_{B} \partial X}\left(c_{B}^{*}\right)>0$.

Now consider the matrix $H$ and recall that $|H|=\frac{\partial^{2} \pi_{A}^{*}}{\partial c_{A} \partial c_{A}} \frac{\partial^{2} \pi_{B}^{*}}{\partial c_{B} \partial c_{B}}-\frac{\partial^{2} \pi_{A}^{*}}{\partial c_{A} \partial c_{B}} \frac{\partial^{2} \pi_{B}^{*}}{\partial c_{B} \partial c_{A}}$. The first term is always positive since $\frac{\partial^{2} \pi_{A}^{*}}{\partial c_{A} \partial c_{A}}<0$ and $\frac{\partial^{2} \pi_{B}^{*}}{\partial c_{B} \partial c_{B}}<0$. The second term is also always positive since $\frac{\partial^{2} \pi_{B}^{*}}{\partial c_{B} \partial c_{A}}>0$ and $\frac{\partial^{2} \pi_{A}^{*}}{\partial c_{A} \partial c_{B}}>0$. Thus, we make a standard stability assumption here that $\left|\frac{\partial^{2} \pi_{i}^{*}}{\partial c_{i} \partial c_{i}}\right|>\left|\frac{\partial^{2} \pi_{i}^{*}}{\partial c_{i} \partial c_{j}}\right|$, which ensures that $|H|>0$. Given the above, the determinants $\left|H_{A}\right|$ and $\left|H_{B}\right|$ are positive, so that $\frac{d c_{A}}{d X}>0$ and $\frac{d c_{B}}{d X}>0$. 


\subsubsection{The effect of $X$ on equilibrium prices and profits}

As for the prices,

$$
\begin{aligned}
& \frac{d p_{A}^{*}}{d X}\left(c_{A}(X), c_{B}(X), X\right)=\frac{\partial p_{A}^{*}}{\partial c_{A}} \frac{d c_{A}}{d X}+\frac{\partial p_{A}^{*}}{\partial c_{B}} \frac{d c_{B}}{d X}+\frac{\partial p_{A}^{*}}{\partial X} \\
& \frac{d p_{B}^{*}}{d X}\left(c_{A}(X), c_{B}(X), X\right)=\frac{\partial p_{B}^{*}}{\partial c_{A}} \frac{d c_{A}}{d X}+\frac{\partial p_{B}^{*}}{\partial c_{B}} \frac{d c_{B}}{d X}+\frac{\partial p_{B}^{*}}{\partial X}
\end{aligned}
$$

since $\frac{\partial p_{A}^{*}}{\partial c_{A}}=\frac{\partial p_{B}^{*}}{\partial c_{A}}=0$, and the remaining terms are strictly positive (as is shown above or can be shown by direct differentiation), $\frac{d p_{A}^{*}}{d X}>0$ and $\frac{d p_{B}^{*}}{d X}>0$.

As for the profits,

$$
\begin{aligned}
& \frac{d \Pi_{A}^{*}}{d X}=\frac{\partial \Pi_{A}^{*}}{\partial c_{A}} \frac{d c_{A}}{d X}+\frac{\partial \Pi_{A}^{*}}{\partial c_{B}} \frac{d c_{B}}{d X}+\frac{\partial \Pi_{A}^{*}}{\partial X}, \\
& \frac{d \Pi_{B}^{*}}{d X}=\frac{\partial \Pi_{B}^{*}}{\partial c_{A}} \frac{d c_{A}}{d X}+\frac{\partial \Pi_{B}^{*}}{\partial c_{B}} \frac{d c_{B}}{d X}+\frac{\partial \Pi_{B}^{*}}{\partial X}
\end{aligned}
$$

by virtue of the envelope theorem, $\frac{\partial \Pi_{A}^{*}}{\partial c_{A}}=0$ and $\frac{\partial \Pi_{B}^{*}}{\partial c_{B}}=0$, and the remaining terms are again strictly positive, so that $\frac{d \Pi_{A}^{*}}{d X}>0$ and $\frac{d \Pi_{B}^{*}}{d X}>0$. 


\section{Working Paper Series}

ISSN 1211-3298

Registration No. (Ministry of Culture): E 19443

Individual researchers, as well as the on-line and printed versions of the CERGE-EI Working Papers (including their dissemination) were supported from the following institutional grants:

- Economic Aspects of EU and EMU Entry [Ekonomické aspekty vstupu do Evropské unie a Evropské měnové unie], No. AVOZ70850503, (2005-2011);

- Economic Impact of European Integration on the Czech Republic [Ekonomické dopady evropské integrace na ČR], No. MSM0021620846, (2005-2011);

Specific research support and/or other grants the researchers/publications benefited from are acknowledged at the beginning of the Paper.

(c) Jiří Střelický, Krešimir Žigić, 2011

All rights reserved. No part of this publication may be reproduced, stored in a retrieval system or transmitted in any form or by any means, electronic, mechanical or photocopying, recording, or otherwise without the prior permission of the publisher.

Published by

Charles University in Prague, Center for Economic Research and Graduate Education (CERGE) and

Economics Institute ASCR, v. v. i. (EI)

CERGE-El, Politických vězňŭ 7, 11121 Prague 1, tel.: +420 224005 153, Czech Republic.

Printed by CERGE-EI, Prague

Subscription: CERGE-EI homepage: http://www.cerge-ei.cz

Phone: + 420224005153

Email: office@cerge-ei.cz

Web: http://www.cerge-ei.cz

Editor: Michal Kejak

Editorial board: Jan Kmenta, Randall Filer, Petr Zemčík

The paper is available online at http://www.cerge-ei.cz/publications/working_papers/.

ISBN 978-80-7343-236-2 (Univerzita Karlova. Centrum pro ekonomický výzkum a doktorské studium)

ISBN 978-80-7344-226-2 (Národohospodářský ústav AV ČR, v. v. i.) 
CERGE-EI

P.O.BOX 882

Politických vězňů 7

11121 Praha 1

Czech Republic http://www.cerge-ei.cz 